\title{
Petrology and Tectonic Setting of Volcanic Rocks in West and South West of Salafchegan, Qom, Iran
}

\author{
Mozhgan Taheri ${ }^{*}$, Afshin Ashja Ardalan1, Mohammad Hashem Emami², \\ Seyed Jamal Shaikh Zakariay ${ }^{3}$ \\ ${ }^{1}$ Department of Geology, North Tehran Branch, Islamic Azad University, Tehran, Iran \\ ${ }^{2}$ Department of Geology, Islamshahr Branch, Islamic Azad University, Tehran, Iran \\ ${ }^{3}$ Department of Geology, Science and Research Branch, Islamic Azad University, Tehran, Iran \\ Email: ${ }^{\star}$ taherisi@yah00.com
}

How to cite this paper: Taheri, M., Ardalan, A.A., Emami, M.H. and Zakariay, S.J.S. (2017) Petrology and Tectonic Setting of Volcanic Rocks in West and South West of Salafchegan, Qom, Iran. Open Journal of Geology, 7, 745-767.

https://doi.org/10.4236/ojg.2017.76050

Received: November 30, 2016

Accepted: May 30, 2017

Published: June 2, 2017

Copyright $\odot 2017$ by authors and Scientific Research Publishing Inc. This work is licensed under the Creative Commons Attribution International License (CC BY 4.0).

http://creativecommons.org/licenses/by/4.0/

\begin{abstract}
In the west and south-west part of Salafchegan near Zavarian village in Iran (in central Iranian volcano plotonic belt), there are some volcanic rocks. Based on petrography and geochemistry findings, these volcanic rocks are Basalt, Andesite, Tracky-Andesite, Trackyte, Latite, Dacite. Tectono-magmatic diagram $\mathrm{Y}$ versus $\mathrm{Zr}$ shows the magmatic arc setting and $\mathrm{Zr} / \mathrm{TiO}_{2}$ versus $\mathrm{Ce} / \mathrm{P}_{2} \mathrm{O}_{5}$ diagram contrasts post colligenal magmatic arcs. The HFSE depletion in the MORB spider diagram shows significant volcanic arc magmatism. The high enrichment of $\mathrm{Eu}, \mathrm{Sr}, \mathrm{Th}, \mathrm{Rb}, \mathrm{Cs}, \mathrm{K}$ (LFS elements) and $\mathrm{Y}, \mathrm{Zr}$, Hf shows negative anomaly and subduction tectonic setting. Based on MORB, Chondrite and primitive mantle spider diagrams, LREE have enriched to HREE in this area. The results of petrography, geochemistry and tectonic setting studies in this area, indicate that neogene magmatism occurred in post colligenal tectonic setting-subduction of Neo-thetise ocean under central Iranian plate in neogen era.
\end{abstract}

\section{Keywords}

Volcanic Rocks, Andesite, Central Iranian Volcano Plotonic Belt, Salafchegan, Iran

\section{Introduction}

The central Iranian volcano-plotonic belt (CIVB), composed on tertiary volcanic and plutonic rock that shows Eocene early Oligocene, middle miocen, Pliocene and quaternary magmatism [1]. The urge Eocene volcanism [2] [3] [4] com- 
posed of basic to acidic calcalkaline magmatic activity in subduction zone [5] [6]. The Oligocene and Miocene plutonic rocks in CIVB introduced to Eocene volcanic rocks [7]. The CIVB is an active volcanic zone in upper cretaceous and Eocene [7]. The CIVB composed of mafic-felsic volcanic and plutonic igneous rocks which are introduced by subvolcanic Oligocene Miocene dykes and stocks [8]-[15]. The country rocks of porphyry copper deposits in CIVB are subvolcano plutonic rocks (Granodiorite, diorite, monzonite, quartz onzonite, ...) occurred in subduction zones and similar to Andian copper deposits [16] [17] [18] [19].

The CIVB has situated between Sanandaj-Sirjan and central Iranian zone. The salafchegan studied area is located at North and North-west of CIVB and shows adakitic and alkaline sequence of colligenal and post colligenal magmas in Pliocene and Quaternary [20]. The magmatic activity in Salafchegan similar to CIVB, is due to subduction of Arabian plate and Neogene magmatism in cretaceous and Oligocene periods [21]-[26] (Figure 1). The host rock of most purified copper which has the position of magmatic arc is shallow intrusive in CIVB. These intrusive have been formed in a magmatic arc and convergent plate margins, which can also be seen in copper mine in Andes [16] [17] [18] [19]. The history of magmatism area in Salafchegan in central Iran represents the subduction of Tethys ocean lithosphere in Eocene below the continent of central Iran which is associated with the rift phenomenon of the Red Sea, Gulf of Aden [27] [28] [29].

After the Oligocene, the rate of convergence increases between Arabic plate and continent of central Iran. The ocean began to be closed and collisions were occurred [6] [22] [23] [24] [25] [26]. Consequently, in Salafchegan region, a number of dextral strike-slip faults with direction of North West-South East were formed as a result of oblique convergence [25] [30].

Traditionally, this area has attracted the attention of geologists by various magmatic and mineral phenomena. In geological map of Emami \& Hajian, 1991, Qom 1,250,000 [31]; igneous masses of region have been studied. Also in geological map of Tafresh (1999), Salafchegan-Khorhe (2000), Alaee Mahabadi and Hajian [32] [33] have studied the mentioned masses.
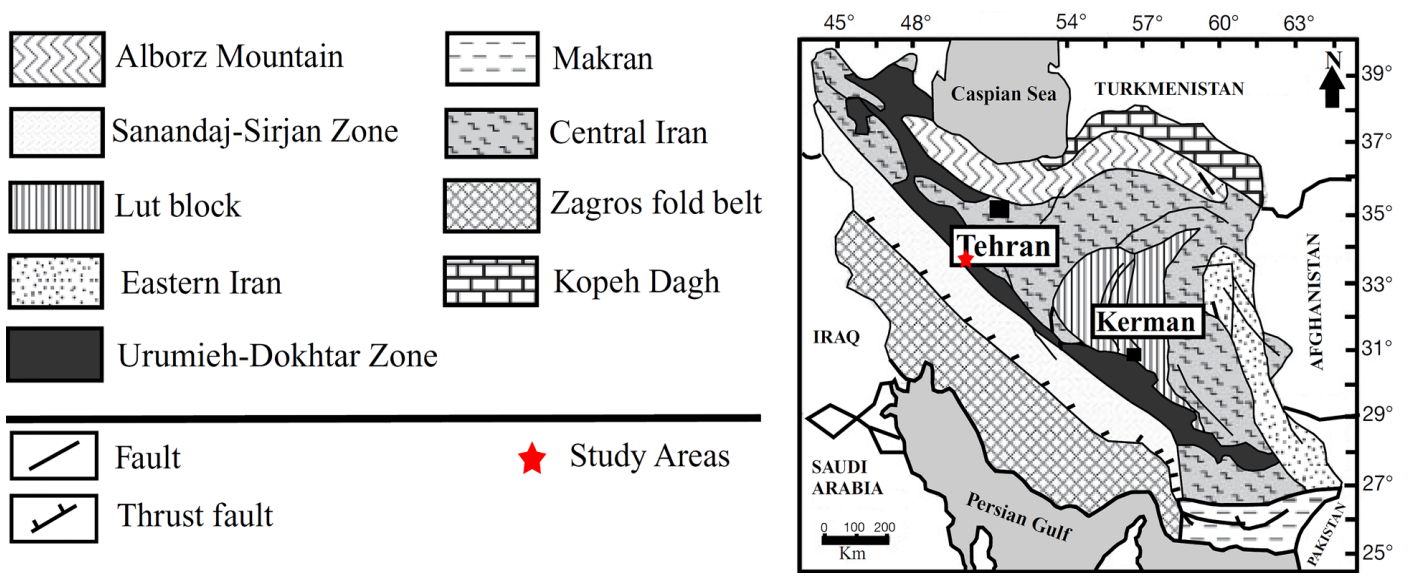

Figure 1. Positions of study area in CIVB magmatic belt (Mirnejad et al., 2013). 
Emami [31] was studied the petrology of Khastak Caldera in Zavarian region in his doctoral thesis titled Geology of Qom-Aran region. Monsef (2001) [34] studied the geochemistry and petrogenesis of Neogene volcanic and semi-volcanic rocks in West of Salafchegan up to North of Delijan. In this study, we attempt to evaluate geochemical features, formation and origin of exterior igneous rocks of the region according to the field data, petrographic studies, and the results of chemical analysis of exposed volcanic rocks.

\section{Research Method}

During the Field Operations, 100 different rock samples were selected with chip sampling method. Samples with minimum weight of $1 \mathrm{~kg}$ placed inside of plastic bags. In order to identify lithological composition and crosscutting relationships, thin microscopic section were prepared and studied. In order geochemical studies, 29 volcanic rocks samples as much as possible unaltered and minimum weathering and secondary streak which covered characteristics of the area rocks were selected and transferred to Geological Survey \& Mineral Explorations of Iran (GSI) laboratory for XRF and ICP-MS analysis. The limit of oxygen index (LOI) was also measured.

\section{Results and Discussion}

\subsection{Region General Geology}

The study area is located in Salafchegan district in $40 \mathrm{~km}$ South West of Qom and near the village of Zvaryan (Figure 1). The study main outcrop was in $34^{\circ} 15^{\prime}$ to $34^{\circ} 40^{\prime}$ North latitude and $50^{\circ} 15^{\prime}$ to $50^{\circ} 28^{\prime}$ East latitude and it is located in an area of about 200 square kilometers.

The Salafchegan region is located in CIVB magmatic zone with direction of North West-South East and composed of Tertiary volcanic intrusive rocks with a length of $1700 \mathrm{~km}$ and an average width of about $150 \mathrm{~km}$. there weren't any outcrops for Precambrian basement in the region and the oldest rock units in Salafchegan area, was sedimentary rocks in Jurassic era (Figure 2). The magmatic activity in this region started in early Eocene and continued until late Eocene [32]. This volcanic complex in the hole of region consists of basaltic to rhyolitic lavas and associated pyroclastic with sedimentary rocks among these layers (Figure 2). The Paleogene volcano-sedimentary sequence has been covered discontinuously with unconformity layers of red and calcareous units [32]. These sequences invaded by granitoid masses with calc-alkaline nature. Shallow intrusive activity continued until the late Miocene and tectonic settings were occurred in Pliocene after the collision. Within the volcanic masses of the area is seen the andesitic dikes to be seen which has cut the volcanic masses that indicates extensive stretching at the end of the volcanic stage.

\subsection{Petrography}

In addition to nomination of initial modal and characterization of Mineralogical and textural thin sections, analysis of events affecting the formation of minerals 

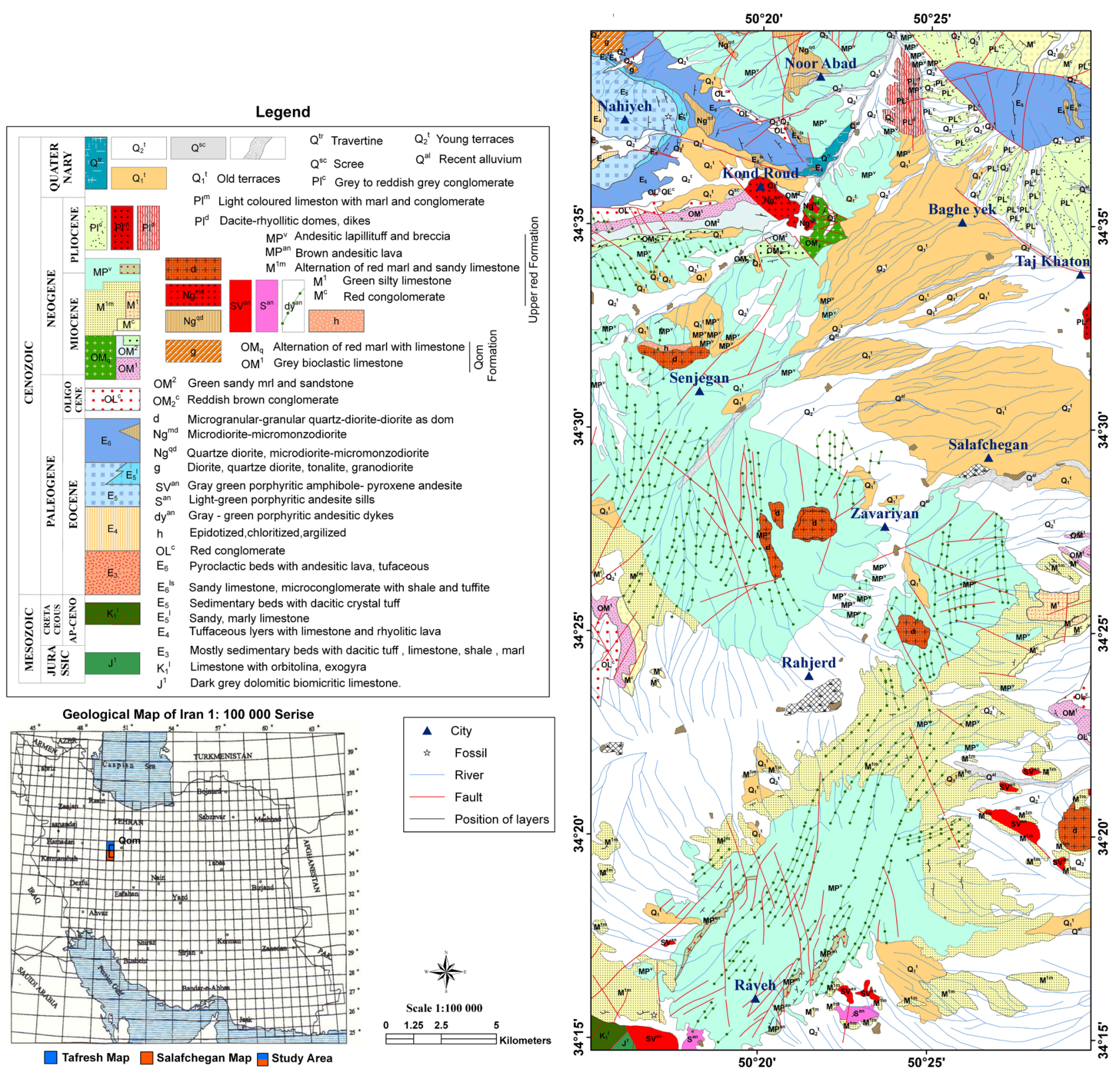

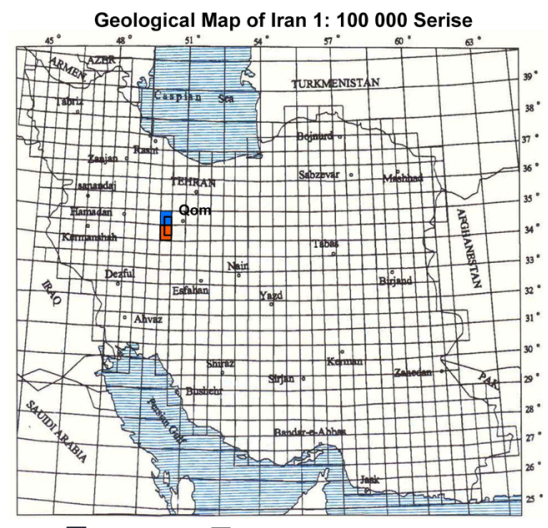

$\square$ Tafresh Map $\square$ Salafchegan Map $\square$ Study Area

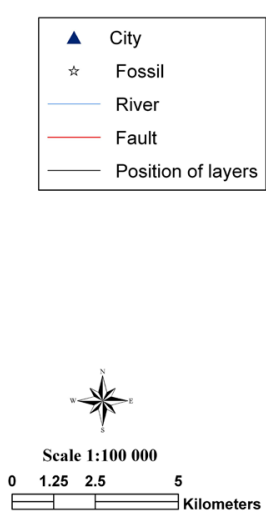

Figure 2. Geology map of study area the resulting Merj (Taheri, 2016) of 1:100,000 Salafchegan-Khorhe geology map (Alaee Mahabadi, 2000) and Tafresh (Hajian, 1999) and field observations.

and identification of texture, mineral relationship (relationship of minerals), reactive margins, Xenoliths and other notable features of were evaluated. Igneous rocks of studied area were divided in two main intrusive and extrusive categories. The volcanic rocks include: olivine basalt, basalt, andesite basalt, andesite, trachyandesite, quartz Latite, Latite, trachyte and dacite and member of intrusive igneous rocks include: diorite, monzonite and quartz monzonite. Based on main purpose of this article, the main volcanic rocks of region are described below.

\subsubsection{Basaltic Lavas}

These rock groups consist of Olivine basalt, basalt, andesite basalt. In terms of texture, composed of porphyric texture with microcrystalline pulp (Figure 3) 
and phenocrysts include of plagioclase, olivine and clinopyroxene. In some samples chlorite and opacities amphibole were observed. Alkali feldspar and quartz can be seen as a rarely sub-grade mineralization. Pennine mineral as result of serve chloritisation were seen in some samples (Figure 4). OPEC minerals were present primarily and secondarily in rocks and rarely tiny cavities which filled with calcite were observed. In some cases, olivine has been serpantnised and formed skeletal structure. Olivine idnegization phenomena startss from margins and fractures and continues to the center of crystals (Figure 5).

\subsubsection{Andesite, Latite, Trachite Lavas}

The most extensive outcrops of volcano rocks in this region are andesitic lavas. These rocks consists of porphyric texture with microlitic pulp stream or microcrystalline, which in some cases formed as a micro porphyric amygdaloid (Figure 6). Disequilibrium textures such as sieve texture (Figure 7) and embayment in these crystals are rarely seen. Presence of sieve texture in plagioclase phenocrysts of andesitic lavas can be due to an imbalance in the magmatic system. Also,

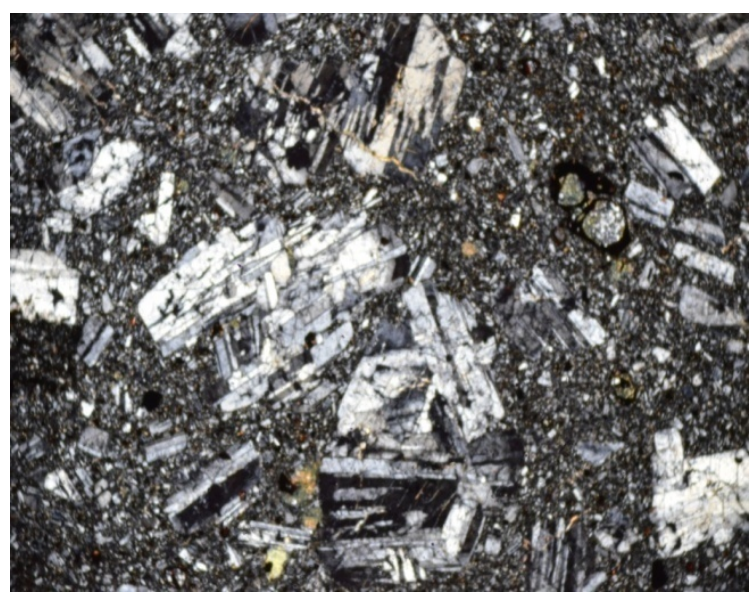

Figure 3. Porphyric texture with microcrystalline pulp (field length: $4.5 \mathrm{~mm}, 40 \mathrm{X})$ (XPL).

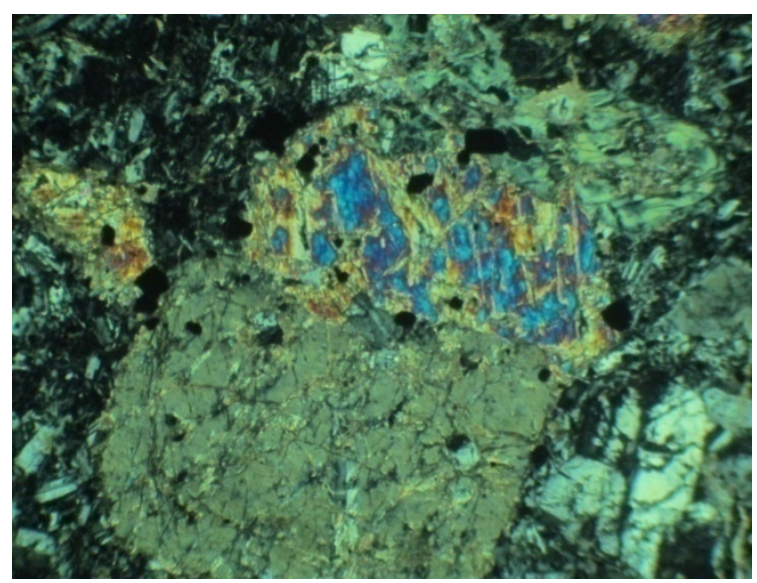

Figure 4. Presence of pennine minerals as result of serve chloritisation in basaltic andesite (field length: $4.5 \mathrm{~mm}$, 40X) (XPL). 


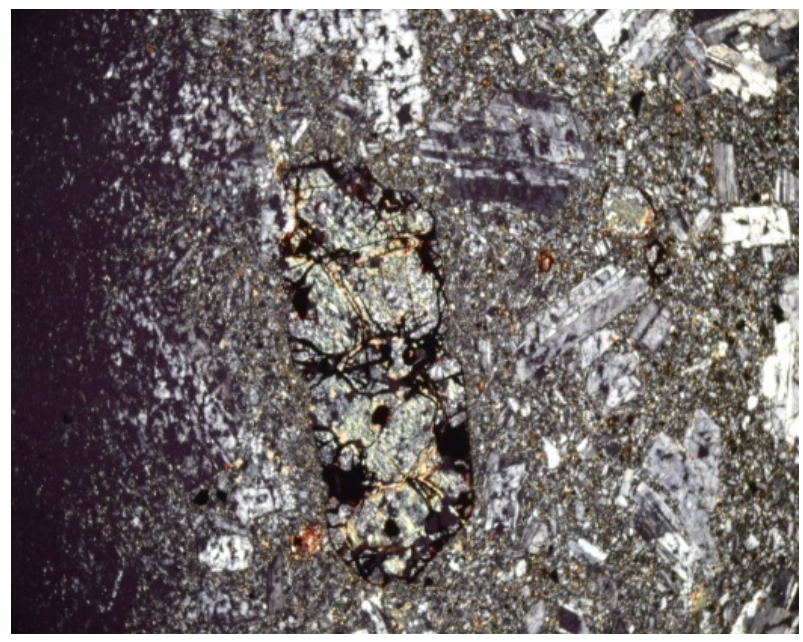

Figure 5. Skeletal structure as result of epastrization of grains of olivine in basalt rocks with serpantinization phenomena (field length: $4.5 \mathrm{~mm}, 40 \mathrm{X}$ ) (XPL).

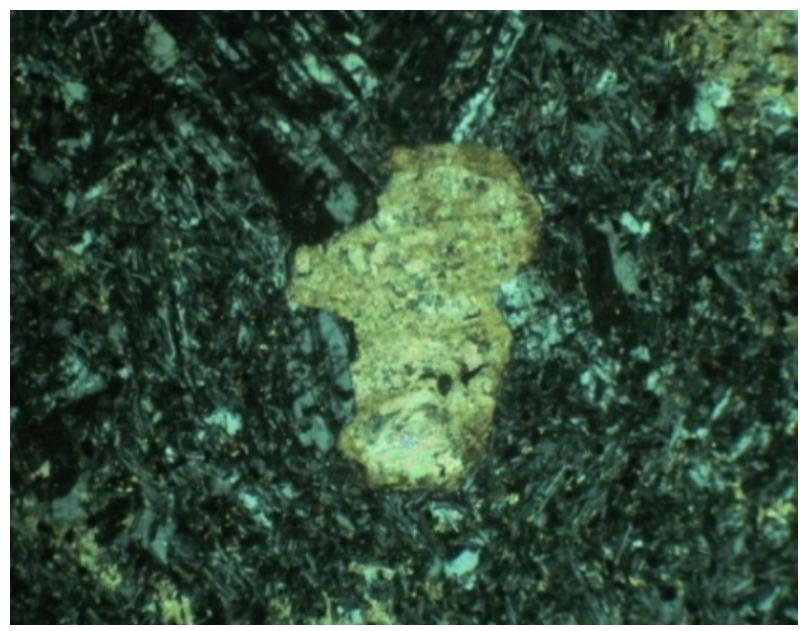

Figure 6. Micro-porphyric amigdual texture with cavities filled by calcite (field length: $4.5 \mathrm{~mm}, 40 \mathrm{X}$ ) (XPL).

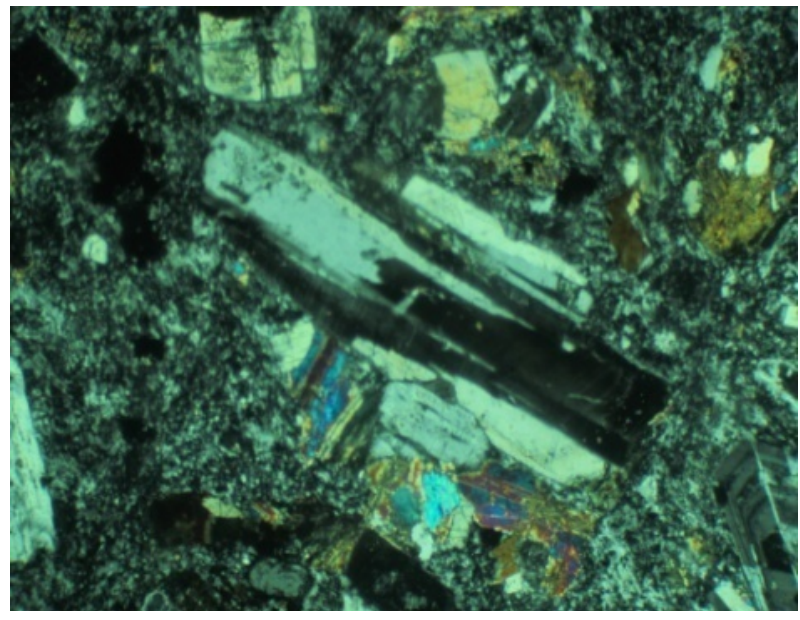

Figure 7. Sieve texture of plagioclase in andesitic rocks (field length: $4.5 \mathrm{~mm}, 40 \mathrm{X})$ (XPL). 
sieve texture in some cases indicates magmatic mixing and sudden fall of pressure and shows unbalanced conditions in magmatic reservoir in general.

Andesitic rocks is consists of fine microliters-oriented plagioclase with some glass between them. Plagioclase which has zoning is the main mineral in these rocks (Figure 8). Twill twinning (Figure 9), crusader twinning and pericline twinning (Figure 10) can be seen in these rocks. Coarse crystals are seen in different sizes in the fine pulps consists of plagioclase, clinopyroxene, burned hornblende (Figure 11), opaque and glass minerals and in some cases alkali feldspar (sanidine) and rarely quartz. Hornblende type of amphibole are mainly altered and replaced with chlorite, calcite and opaque minerals. Presence of rim reaction in amphibole crystals depends on rate of ascent of magma. Secondary minerals include sericite, epidote, silica, iron oxide and hydroxide mineral.

Phenocrysts are visible in Latite of plagioclase, potassium feldspar, approximately in the same values in the crystallized in fine to glasses. Plagioclase is the most abundant Connie Stone which is largely altered. Amphibole macro crystals were seen as automorphe and sub-automorphe with burned margins are often seen as chloritisate, opacitiesate, epidotisate and carbonatizate. The dominant

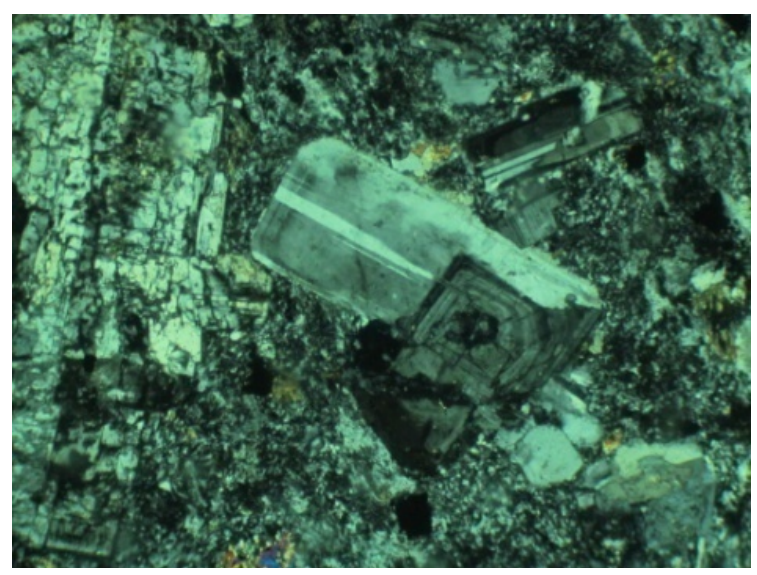

Figure 8. Zoning of plagioclase phenocrysts in the andesite (field length: $4.5 \mathrm{~mm}, 40 \mathrm{X})$ (XPL).

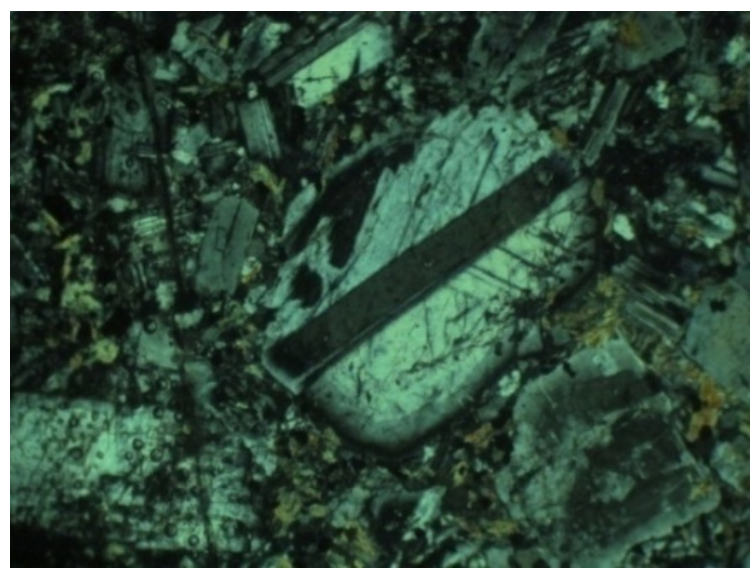

Figure 9. Twill twining of plagioclase in andesite (field length: $4.5 \mathrm{~mm}, 40 \mathrm{X})$ (XPL). 


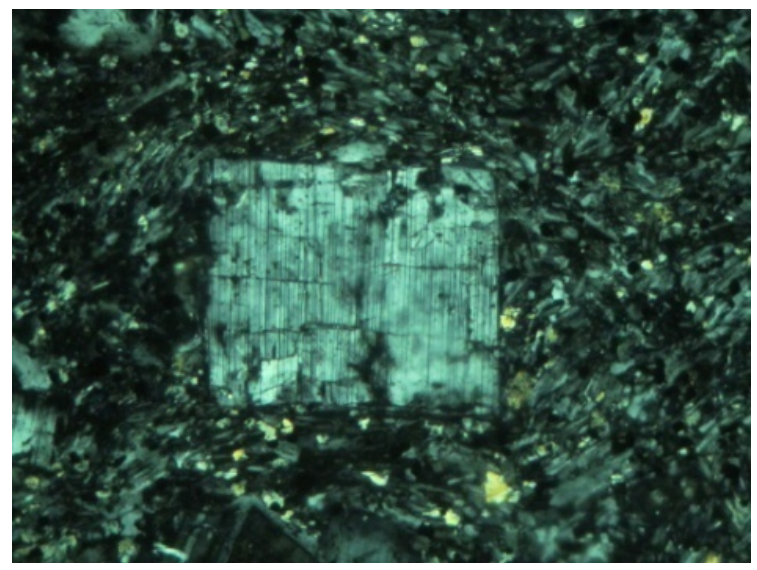

Figure 10. Pericline twinning of plagioclase in andesite (field length: $1.8 \mathrm{~mm}, 10 \mathrm{X}$ ) (XPL).

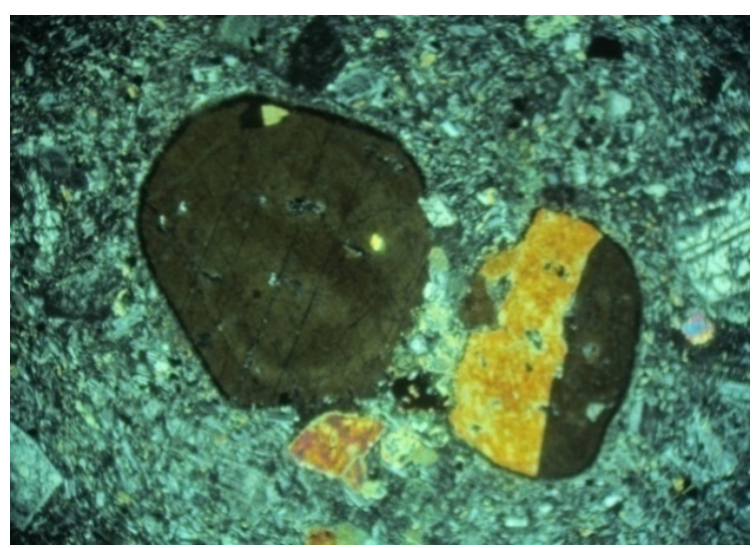

Figure 11. Amphibole phenocrysts in the andesite with burned margins (field length: $4.5 \mathrm{~mm}, 40 \mathrm{X}$ ) (XPL).

process in region hornblendes is apasytisation of hornblendes. In some samples, hornblendes have maintained their hexagonal or diamond shape but completely oxidized. This phenomenon is result of formaion OPEC minerals which seems to be magnetite and hematite. Alkali feldspar is formed as macro crystals up to $40 \%$ of phenocrysts. Minor minerals consist of epidote, chlorite, epic minerals and quartz which includes about 2 percent of pulp. Micro enclaves with granitic composition can be seen in a number of andesitic sections (Figure 12).

\subsubsection{Dacitic Lavas}

Plagioclase, alkali feldspar, quartz amphibole have created the mineralogical composition of the rocks. Plagioclase as the most abundant phenocrysts has polysynthetic twinning and clear zoning combination. Presence of zoning combination and consequently regional blackouts represent complex process in the liquid-crystal interface during rapid cooling in lower temperature [35]. In the other hand, the zoning of plagioclase indicates an imbalance during the crystallization and solidification which can be the result of changes in crystal local conditions like thermal turbulence caused by the entry fresh magma in crystallization of magma chamber, rapid decrease in temperature and changes in the water 
vapor pressure and dissolved gases during crystallization of plagioclase in the magma [36].

Amphibole, is often seen as shaped crystals (Figure 13) to hypidiomorphic in fine pulps. In some amphibole crystals, burned margins are observed. In most cases these burned margins are the result of oxidation reactions which are caused by heat from sudden pressure drop in magma during ascent.

Alkali feldspar is another minerals of these rocks which seen mostly as fine crystals in stone pulp and it is difficult to be diagnosed. Epic minerals were seen primarily and secondarily in stone pulp. Secondary epic minerals mostly formed as result of hornblende alteration and they are seen in the margin and center of minerals.

Porphyric texture with micro crystal pulp (Figure 14), is the most abundant texture of region dacite rocks. Plagioclase and alkali feldspar, shaped feldspars with eroded margins, are the most important coarse crystals. In the other hand, plagioclase coarse crystals were seen with swinging zoning, which it is the sign of crystallization in unstable physicochemical conditions. Erosion of a solid phase in a fluid phase is a sign of fluid saturation which means that the initial

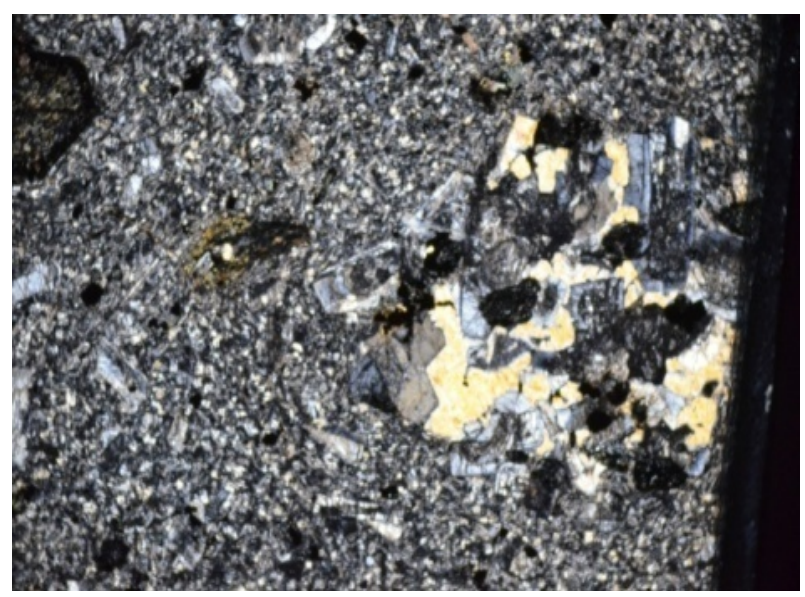

Figure 12. Granite micro enclaves in the andesitic rocks (field length: $4.5 \mathrm{~mm}, 40 \mathrm{X})$ (XPL).

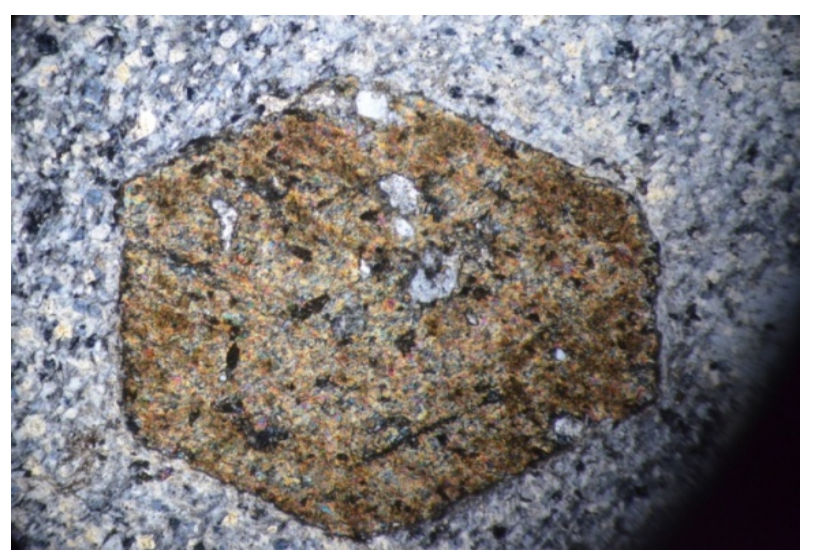

Figure 13. Amphibole phenocrysts in dacite (field length: 1.8 $\mathrm{mm}, 100 \mathrm{X})$ (XPL). 
phase, which has crystallized in fluid due to saturation, is undergoing liquidation due to changing circumstances. Sudden change in temperature, pressure, oxygen fugacity, vapor pressure, digestion stones stuck inside or mixing with other liquid cause changing in the composition of the fluid [36].

\subsection{Geochemistry}

\subsubsection{Classification Extrusive Igneous Rocks and Magma}

In this study we used diagram presented by Cox et al. diagram [37] for nomination of volcanic rocks. According to the results the rocks of region are within the basalt, andesite basalt, andesite and dacite (Figure 15) with sub-alkaline nature and acid intermediate composition. According to the results of geochemical analysis of samples (Table 1), these samples based on the Winchester \& Floyd

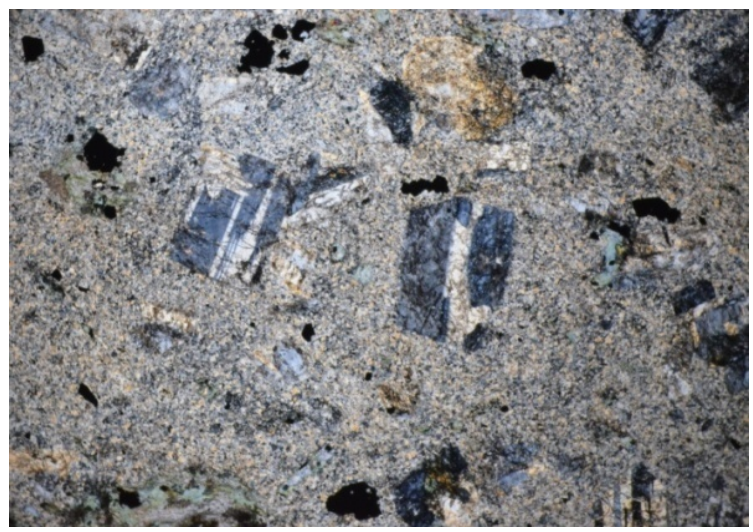

Figure 14. Porphyric texture in dacite (field length: 4.5 $\mathrm{mm}, 40 \mathrm{X})$ (XPL).

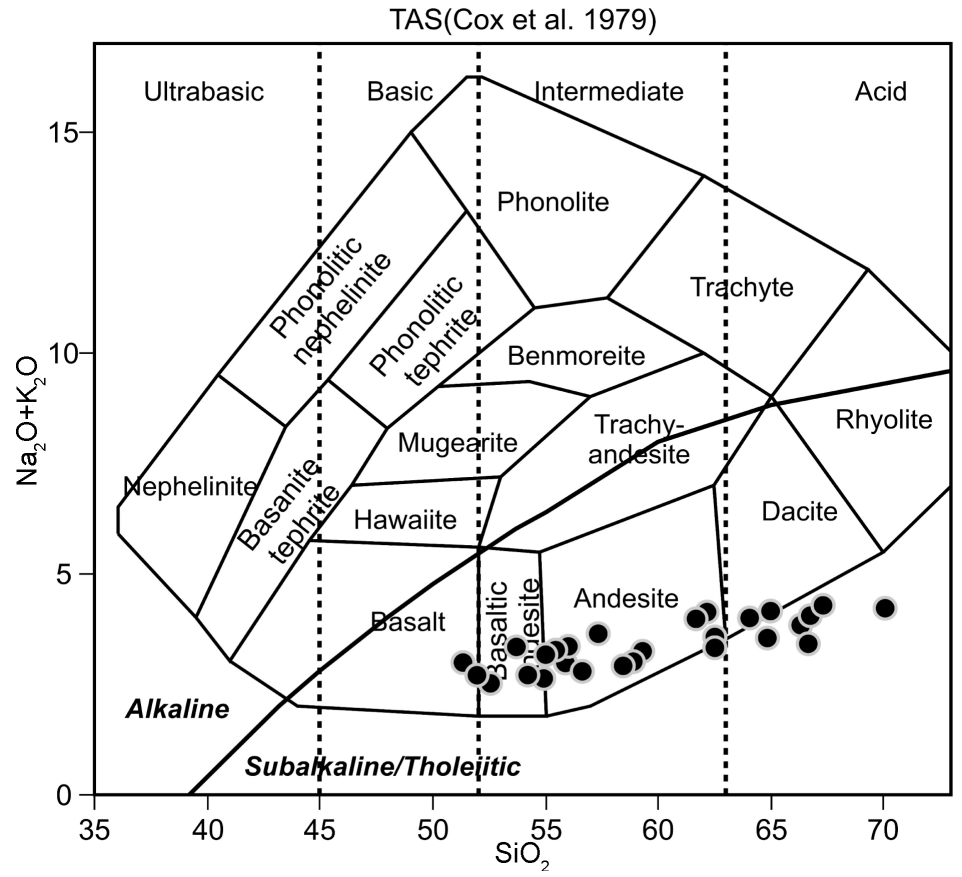

Figure 15. Location of studied volcanic rocks in volcanic rocks nomination charts based on TAS method (Cox et al., 1979). 
Table 1. The result of samples analyses from the studied area by XRF and ICP-OES methods (major elements in \% and trace elements in ppm).

\begin{tabular}{|c|c|c|c|c|c|c|c|c|c|c|c|c|c|c|c|c|c|}
\hline Sample No. & $\mathrm{SiO}_{2}$ & $\mathrm{Al}_{2} \mathrm{O}_{3}$ & $\mathrm{Fe}_{2} \mathrm{O}_{3}$ & $\mathrm{CaO}$ & $\mathrm{MgO}$ & $\mathrm{Na}_{2} \mathrm{O}$ & $\mathrm{K}_{2} \mathrm{O} \quad \mathrm{I}$ & $\mathrm{MnO}$ & $\mathrm{TiO}_{2}$ & $\mathrm{P}_{2} \mathrm{O}_{5}$ & Total & $\mathrm{SrO}$ & $\mathrm{Ag}$ & As & B & $\mathrm{Ba}$ & $\mathrm{Be}$ \\
\hline M11 & 52.1 & 18.8 & 8.8 & 8.2 & 5.0 & 2.3 & 0.9 & 0.1 & 0.5 & 0.1 & 96.8 & 0.1 & 0.25 & 3.04 & 7.95 & 94 & 0.67 \\
\hline M16 & 53.9 & 18.3 & 6.4 & 7.2 & 4.1 & 2.5 & 1.0 & 0.1 & 0.4 & 0.2 & 94.1 & 0.1 & 0.20 & 4.01 & 8.91 & 144 & 0.86 \\
\hline M20 & 56.6 & 19.0 & 7.7 & 6.1 & 3.6 & 2.2 & 0.6 & 0.2 & 0.6 & 0.2 & 96.8 & 0.1 & 0.18 & 9.65 & 7.36 & 193 & 1.03 \\
\hline M22 & 53.8 & 17.7 & 9.2 & 7.7 & 2.9 & 1.9 & 0.8 & 0.2 & 0.8 & 0.2 & 95.2 & 0.1 & 0.41 & 6.72 & 8.30 & 373 & 1.15 \\
\hline M25 & 53.5 & 19.7 & 8.7 & 8.3 & 3.7 & 2.1 & 0.4 & 0.2 & 0.5 & 0.2 & 97.3 & 0.1 & 0.41 & 3.19 & 9.63 & 353 & 1.35 \\
\hline M26 & 54.3 & 19.6 & 8.5 & 7.1 & 3.2 & 2.7 & 0.6 & 0.2 & 0.6 & 0.2 & 97 & 0.1 & 0.28 & 4.38 & 9.46 & 145 & 0.88 \\
\hline M31 & 50.3 & 18.6 & 10.6 & 8.1 & 5.4 & 2.1 & 0.3 & 0.3 & 0.8 & 0.2 & 96.7 & 0.09 & 0.20 & 4.01 & 8.87 & 214 & 0.98 \\
\hline M33 & 60.3 & 17.2 & 6.3 & 5.8 & 2.4 & 2.1 & 1.4 & 0.2 & 0.4 & 0.2 & 96.3 & 0.08 & 0.17 & 0.56 & 9.13 & 143 & 0.88 \\
\hline M34 & 60.0 & 17.1 & 7.5 & 5.0 & 2.4 & 2.9 & 1.1 & 0.2 & 0.8 & 0.2 & 97.2 & 0.09 & 0.11 & 4.32 & 7.70 & 530 & 1.24 \\
\hline M37 & 61.3 & 17.3 & 7.4 & 5.6 & 1.6 & 2.9 & 1.2 & 0.2 & 0.8 & 0.2 & 98.5 & 0.09 & 0.24 & 5.19 & 9.34 & 509 & 1.71 \\
\hline M40 & 49.9 & 21.0 & 10.0 & 8.8 & 3.2 & 2.5 & 0.4 & 0.2 & 1.0 & 0.2 & 97.2 & 0.1 & 0.16 & 0.69 & 7.34 & 384 & 1.60 \\
\hline M41 & 65.5 & 16.0 & 5.0 & 3.7 & 2.1 & 3.0 & 1.2 & 0.2 & 0.4 & 0.2 & 97.3 & 0.09 & 0.20 & 10.91 & 9.80 & 167 & 1.02 \\
\hline M43 & 65.0 & 16.3 & 5.2 & 4.1 & 2.1 & 2.9 & 1.1 & 0.2 & 0.4 & 0.2 & 97.5 & 0.09 & $<0.1$ & 6.82 & 7.92 & 355 & 1.21 \\
\hline M48 & 54.0 & 18.9 & 9.9 & 7.1 & 3.2 & 2.6 & 0.2 & 0.2 & 0.5 & 0.2 & 96.8 & 0.1 & 0.19 & 3.26 & 6.12 & 213 & 1.01 \\
\hline M5 & 55.5 & 19.0 & 8.6 & 7.4 & 4.1 & 2.5 & 0.2 & 0.1 & 0.5 & 0.2 & 98.1 & 0.1 & 0.22 & 10.75 & 11.24 & 160 & 0.92 \\
\hline M50 & 62.7 & 17.3 & 5.8 & 5.2 & 2.1 & 3.0 & 0.9 & 0.1 & 0.4 & 0.2 & 97.7 & 0.1 & $<0.1$ & 6.24 & 8.32 & 112 & 0.88 \\
\hline M53 & 53.5 & 19.0 & 10.0 & 7.4 & 3.3 & 2.8 & 0.3 & 0.2 & 0.6 & 0.1 & 97.2 & 01 & 0.45 & 1.87 & 6.05 & 351 & 1.14 \\
\hline M55 & 58.0 & 18.9 & 7.5 & 7.7 & 2.7 & 2.4 & 0.5 & 0.1 & 0.5 & 0.2 & 98.5 & 0.1 & 0.23 & 3.61 & 10.10 & 138 & 0.87 \\
\hline M60 & 58.0 & 19.1 & 7.3 & 6.8 & 2.9 & 2.6 & 0.5 & 0.1 & 0.5 & 0.1 & 97.9 & 0.1 & 0.39 & 1.40 & 7.98 & 120 & 0.83 \\
\hline M63 & 65.2 & 17.1 & 0.1 & 4.6 & 1.6 & 3.1 & 0.3 & 4.9 & 0.3 & 0.2 & 97.4 & 0.09 & 0.16 & 4.96 & 6.50 & 179 & 0.96 \\
\hline M69 & 63.0 & 17.5 & 5.4 & 5.1 & 1.9 & 3.0 & 0.5 & 0.1 & 0.4 & 0.2 & 97.1 & 0.1 & 0.28 & 5.14 & 6.25 & 141 & 1.01 \\
\hline M72 & 61.3 & 17.4 & 6.6 & 5.6 & 2.9 & 2.8 & 0.5 & 0.2 & 0.5 & 0.2 & 98 & 0.1 & 0.20 & 1.47 & 8.54 & 123 & 0.78 \\
\hline M75 & 69.1 & 17.2 & 0.1 & 3.7 & 1.0 & 3.3 & 1.0 & 2.5 & 0.2 & 0.2 & 98.3 & 0.09 & 0.11 & 5.56 & 6.80 & 125 & 1.04 \\
\hline M76 & 63.7 & 17.3 & 5.4 & 5.5 & 2.0 & 2.8 & 0.7 & 0.1 & 0.4 & 0.2 & 98.1 & 0.1 & 0.24 & 5.95 & 6.13 & 132 & 1.12 \\
\hline M78 & 65.0 & 16.8 & 5.0 & 5.0 & 1.6 & 3.0 & 0.8 & 0.2 & 0.4 & 0.2 & 98 & 0.1 & 0.21 & $<0.5$ & 6.90 & 207 & 1.10 \\
\hline M8 & 64.4 & 18.1 & 4.4 & 6.1 & 1.4 & 3.3 & 0.8 & 0.2 & 0.4 & 0.2 & 99.3 & 0.09 & 0.18 & 1.90 & 6.03 & 141 & 0.98 \\
\hline M82 & 52.2 & 16.8 & 10.8 & 7.1 & 5.7 & 2.4 & 0.1 & 0.2 & 0.9 & 0.1 & 96.3 & 0.09 & 0.17 & 1.70 & 7.20 & 256 & 1.13 \\
\hline M86 & 50.2 & 18.5 & 10.7 & 8.0 & 4.4 & 1.8 & 0.4 & 0.2 & 1.0 & 0.2 & 95.4 & 0.1 & 0.35 & 0.96 & 8.88 & 127 & 0.93 \\
\hline M93 & 54.7 & 21.1 & 8.1 & 8.1 & 2.4 & 2.4 & 0.8 & 0.2 & 0.7 & 0.2 & 98.7 & 0.09 & 0.13 & 4.53 & 6.83 & 348 & 1.14 \\
\hline Sample No. & $\mathrm{Bi}$ & $\mathrm{Cd}$ & $\mathrm{Ce}$ & Co & $\mathrm{Cr}$ & Cs & $\mathrm{Cu}$ & 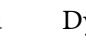 & & Er & $\mathrm{Eu}$ & $\mathrm{Ga}$ & Gd & Ge & $\mathrm{Hf}$ & $\mathrm{Hg}$ & Ho \\
\hline M11 & 0.4 & 0.96 & 14.66 & 18.37 & 54.32 & 5.68 & 2.43 & 3 & & 22 & 0.90 & 18.24 & 4.29 & 2.21 & 1.82 & 0.03 & 0.47 \\
\hline M16 & 0.4 & 1.18 & 19.26 & 14.12 & 51.23 & 8.41 & 1.14 & 2.5 & & 47 & 0.98 & 15.75 & 3.95 & 3.27 & 2.58 & 0.06 & 0.44 \\
\hline M20 & 0.69 & 1.29 & 30.17 & 14.05 & 75.42 & 9.88 & 80.16 & 2.4 & & .13 & 1.22 & 20.19 & 5.71 & 3.37 & 2.63 & 0.04 & 0.47 \\
\hline M22 & 0.4 & 1.44 & 39.21 & 17.07 & 15.99 & $\begin{array}{ll}9 & 11.25\end{array}$ & 55.81 & 3.5 & & .74 & 1.29 & 18.24 & 5.40 & 3.81 & 2.87 & 0.05 & 0.50 \\
\hline M25 & 0.5 & 1.42 & 19.55 & 19.84 & 39.99 & $9 \quad 11.00$ & 25.57 & 2.6 & & .75 & 0.85 & 14.90 & 4.04 & 3.83 & 2.89 & 0.03 & 0.44 \\
\hline M26 & 0.4 & 1.36 & 18.26 & 14.25 & 19.57 & 9.88 & 14.38 & 2.6 & & .34 & 0.93 & 15.66 & 5.05 & 3.14 & 2.81 & 0.02 & 0.42 \\
\hline M31 & 0.4 & 1.19 & 19.16 & 25.81 & 59.42 & 8.35 & 35.47 & 3.5 & & .81 & 1.19 & 17.46 & 8.52 & 3.18 & 2.49 & 0.05 & 0.36 \\
\hline M33 & 0.4 & 1.23 & 31.41 & 11.14 & 32.93 & 9.76 & 5.70 & 2.8 & & .72 & 0.84 & 13.20 & 3.20 & 2.89 & 2.33 & 0.03 & 0.39 \\
\hline M34 & 0.4 & 1.30 & 43.16 & 8.96 & 13.65 & $5 \quad 10.22$ & 8.52 & 6.8 & & .76 & 1.52 & 19.57 & 10.13 & 3.23 & 2.57 & 0.05 & 0.65 \\
\hline
\end{tabular}




\section{Continued}

\begin{tabular}{|c|c|c|c|c|c|c|c|c|c|c|c|c|c|c|c|c|}
\hline M37 & 0.4 & 0.99 & 38.96 & 7.44 & 29.39 & 7.24 & 10.17 & 5.63 & 3.66 & 1.45 & 20.98 & 9.42 & 2.29 & 1.99 & 0.05 & 0.48 \\
\hline M40 & 0.4 & 1.51 & 30.08 & 23.00 & 59.08 & 11.16 & 9.73 & 4.14 & 3.30 & 1.42 & 20.39 & 9.15 & 3.41 & 2.87 & 0.02 & 0.34 \\
\hline M41 & 0.4 & 1.01 & 41.46 & 4.18 & 61.27 & 8.09 & 4.41 & 4.55 & 3.62 & 0.76 & 14.94 & 3.54 & 2.65 & 2.08 & 0.05 & 0.33 \\
\hline M43 & 0.4 & 1.07 & 31.95 & 5.71 & 33.13 & 7.71 & 2.79 & 3.54 & 3.43 & 0.69 & 17.73 & 3.19 & 2.39 & 2.13 & 0.05 & 0.33 \\
\hline M48 & 0.3 & 1.61 & 26.12 & 18.31 & 34.03 & 12.40 & 32.89 & 3.46 & 2.13 & 1.76 & 21.60 & 5.65 & 4.17 & 3.18 & 0.05 & 0.49 \\
\hline M5 & 0.4 & 1.11 & 19.61 & 17.44 & 76.12 & 8.05 & 105 & 1.22 & 1.73 & 1.07 & 19.85 & 6.21 & 2.79 & 2.30 & 0.04 & 0.52 \\
\hline M50 & 0.3 & 0.88 & 27.47 & 7.77 & 28.33 & 7.39 & 5.44 & 2.30 & 1.00 & 1.07 & 16.19 & 4.09 & 2.04 & 1.69 & 0.04 & 0.31 \\
\hline M53 & 0.3 & 1.50 & 25.37 & 21.25 & 42.77 & 11.21 & 32.96 & 2.88 & 2.83 & 1.49 & 20.00 & 7.62 & 3.66 & 3.03 & 0.05 & 0.42 \\
\hline M55 & 0.3 & 1.12 & 19.90 & 4.61 & 44.62 & 9.15 & 3.31 & 2.11 & 1.45 & 0.90 & 20.09 & 4.11 & 2.79 & 2.29 & 0.03 & 0.28 \\
\hline M60 & 0.4 & 1.10 & 18.70 & 12.45 & 36.16 & 9.61 & 15.64 & 1.90 & 1.64 & 0.91 & 17.17 & 3.60 & 2.68 & 2.33 & 0.05 & 0.33 \\
\hline M63 & 0.3 & 0.86 & 26.35 & 6.48 & 9.78 & 6.36 & 1.46 & 2.17 & 2.35 & 0.78 & 16.55 & 2.47 & 2.40 & 1.91 & 0.03 & 0.19 \\
\hline M69 & 0.3 & 0.99 & 26.56 & 5.40 & 35.33 & 7.71 & 16.57 & 2.48 & 2.36 & 0.99 & 20.98 & 4.36 & 2.49 & 2.20 & 0.03 & 0.27 \\
\hline M72 & 0.3 & 0.89 & 29.14 & 15.30 & 65.47 & 8.13 & 19.30 & 3.83 & 1.29 & 1.27 & 17.73 & 5.04 & 2.29 & 2.15 & 0.03 & 0.28 \\
\hline M75 & 0.4 & 0.37 & 29.40 & 2.79 & 24.51 & 3.75 & 0.87 & 1.91 & 0.71 & 0.65 & 15.91 & 1.59 & 1.02 & 0.91 & 0.03 & 0.20 \\
\hline M76 & 0.4 & 0.77 & 31.99 & 13.25 & 63.60 & 6.30 & 12.01 & 4.01 & $<0.5$ & 1.44 & 22.90 & 5.47 & 2.30 & 1.81 & 0.03 & 0.30 \\
\hline M78 & 0.3 & 0.74 & 27.09 & 6.25 & 28.45 & 6.92 & 6.68 & 3.36 & 1.39 & 1.35 & 17.17 & 4.25 & 1.93 & 1.77 & 0.03 & 0.20 \\
\hline M8 & 1.37 & 0.62 & 22.73 & 9.83 & 42.02 & 5.07 & 10.15 & 2.99 & 2.78 & 0.91 & 17.33 & 3.90 & 1.20 & 1.20 & 0.02 & 0.46 \\
\hline M82 & 0.4 & 1.28 & 32.23 & 25.37 & 35.65 & 11.42 & 5.71 & 4.70 & 3.53 & 1.24 & 19.13 & 7.89 & 3.29 & 3.05 & 0.03 & 0.32 \\
\hline M86 & 0.3 & 1.20 & 34.94 & 22.18 & 68.01 & 11.28 & 16.09 & 5.74 & 2.50 & 2.29 & 20.75 & 10.14 & 3.23 & 3.03 & 0.03 & 0.36 \\
\hline M93 & 0.51 & 0.87 & 38.55 & 18.83 & 22.20 & 7.84 & 13.43 & 5.81 & 2.90 & 1.35 & 16.54 & 8.94 & 2.63 & 2.06 & 0.04 & 0.40 \\
\hline Sample No. & In & $\mathrm{La}$ & $\mathrm{Li}$ & $\mathrm{Lu}$ & $\mathrm{Mn}$ & Mo & $\mathrm{Nb}$ & $\mathrm{Nd}$ & $\mathrm{Ni}$ & $\mathrm{P}$ & $\mathrm{Pb}$ & $\operatorname{Pr}$ & $\mathrm{Rb}$ & $S$ & $\mathrm{Sb}$ & $\mathrm{Sc}$ \\
\hline M11 & 0.4 & 9.38 & 8.49 & 0.64 & 654 & 2.50 & 17.09 & 24.08 & 10.66 & 522 & 7.24 & 2.45 & 200 & 101 & 1.08 & 20.43 \\
\hline M16 & 0.4 & 10.05 & 8.66 & 0.73 & 650 & 1.47 & 16.48 & 25.67 & 7.66 & 744 & 2.68 & 2.73 & 216 & 94.92 & 0.58 & 15.54 \\
\hline M20 & 0.4 & 15.03 & 18.50 & 0.62 & 887 & 3.67 & 21.04 & 32.23 & 6.99 & 749 & 8.01 & 3.59 & 223 & 28.42 & 0.59 & 22.94 \\
\hline M22 & 0.57 & 18.62 & 19.43 & 0.70 & 1045 & 2.41 & 22.05 & 36.58 & 5.38 & 795 & 57.46 & 3.61 & 194 & 65.90 & 0.62 & 23.02 \\
\hline M25 & 0.52 & 10.43 & 14.53 & 0.63 & 933 & 2.49 & 16.37 & 27.86 & 12.31 & 744 & 7.17 & 2.72 & 179 & 56.38 & 0.58 & 13.64 \\
\hline M26 & 0.70 & 10.29 & 14.94 & 0.61 & 1289 & 1.62 & 16.57 & 27.16 & 11.31 & 757 & 6.07 & 3.35 & 212 & 75.43 & 0.63 & 14.10 \\
\hline M31 & 0.62 & 9.85 & 16.10 & 0.77 & 1198 & 0.96 & 23.31 & 34.89 & 12.82 & 612 & 3.86 & 3.41 & 156 & 133 & 0.59 & 29.80 \\
\hline M33 & 0.57 & 19.42 & 17.59 & 0.61 & 1004 & 0.70 & 13.55 & 26.49 & 4.64 & 942 & 5.87 & 3.13 & 178 & 34.36 & 0.51 & 9.60 \\
\hline M34 & 0.66 & 23.09 & 12.35 & 0.75 & 1094 & 1.29 & 26.87 & 45.64 & 3.53 & 1111 & 11.13 & 4.72 & 180 & 39.36 & 0.50 & 18.95 \\
\hline M37 & 0.61 & 21.54 & 13.63 & 0.63 & 999 & 3.18 & 25.15 & 42.90 & 2.21 & 1160 & 6.64 & 4.06 & 156 & 47.82 & 0.51 & 20.43 \\
\hline M40 & 0.4 & 12.17 & 12.36 & 0.77 & 992 & 1.78 & 27.32 & 38.22 & 8.29 & 796 & 1.31 & 4.54 & 192 & 4.09 & 0.50 & 21.89 \\
\hline M41 & 0.75 & 24.04 & 13.73 & 0.56 & 1219 & 2.23 & 12.24 & 28.68 & 2.00 & 1034 & 13.19 & 3.83 & 153 & 10.41 & 0.51 & 7.52 \\
\hline M43 & 0.56 & 21.14 & 14.44 & 0.43 & 1024 & 2.54 & 11.37 & 26.39 & 2.86 & 1022 & 14.70 & 2.01 & 127 & 12.49 & $<0.5$ & 6.65 \\
\hline M48 & 0.74 & 13.60 & 22.14 & 0.92 & 1202 & 1.31 & 17.68 & 30.56 & 5.63 & 738 & 7.90 & 4.16 & 305 & 64.38 & 0.55 & 16.89 \\
\hline M5 & 0.4 & 10.83 & 15.19 & 0.74 & 680 & 3.44 & 17.69 & 25.06 & 13.64 & 598 & 4.44 & 3.30 & 210 & 116 & 0.62 & 22.71 \\
\hline M50 & 0.4 & 16.74 & 18.14 & 0.60 & 911 & 0.89 & 13.72 & 27.77 & 5.07 & 945 & $<0.5$ & 3.28 & 267 & 39.21 & 0.57 & 8.90 \\
\hline M53 & 0.67 & 13.45 & 16.59 & 0.74 & 1147 & 2.95 & 19.31 & 33.47 & 3.79 & 622 & 1.40 & 5.06 & 297 & 32.33 & 0.57 & 20.47 \\
\hline M55 & 0.4 & 11.01 & 11.55 & 0.83 & 562 & 1.32 & 14.43 & 25.70 & 2.14 & 672 & 1.89 & 3.68 & 237 & 62.10 & 0.64 & 14.37 \\
\hline M60 & 0.4 & 11.20 & 12.29 & 0.65 & 747 & 1.26 & 12.75 & 24.75 & 4.64 & 627 & 23.88 & 3.08 & 219 & 75.91 & 0.52 & 12.47 \\
\hline M63 & 0.4 & 15.30 & 13.64 & 0.29 & 380 & 3.53 & 10.04 & 23.44 & 2.91 & 807 & 3.67 & 2.64 & 184 & 26.87 & $<0.5$ & 6.18 \\
\hline
\end{tabular}


M. Taheri et al.

Continued

\begin{tabular}{|c|c|c|c|c|c|c|c|c|c|c|c|c|c|c|c|c|c|c|c|}
\hline M69 & 0.4 & 18.2 & \multicolumn{2}{|c|}{19.27} & 0.59 & 707 & $<0.5$ & 14.72 & \multicolumn{2}{|c|}{29.78} & 4.76 & 958 & 8.52 & 3.50 & 336 & \multicolumn{2}{|c|}{60.40} & 0.52 & 9.66 \\
\hline M72 & 0.6 & 16.6 & 34. & & 0.53 & 1199 & 4.51 & 17.47 & 33. & & 6.34 & 922 & 13.42 & 3.96 & 302 & & .40 & 0.57 & 13.40 \\
\hline M75 & 0.4 & 22.0 & 21. & & 0.14 & 594 & 2.72 & 6.87 & 21. & & 1.30 & 597 & 1.44 & 1.92 & 305 & & .50 & $<0.5$ & 2.32 \\
\hline M76 & 0.69 & 20.9 & 26. & & 0.68 & 1209 & 2.01 & 16.46 & 36. & & 3.10 & 910 & 10.73 & 4.23 & 431 & & .18 & 0.56 & 11.47 \\
\hline M78 & 0.58 & 17.0 & 22. & & 0.39 & 1131 & 2.38 & 13.14 & 31. & & 1.74 & 888 & 5.11 & 2.95 & 282 & & 74 & $<0.5$ & 8.06 \\
\hline M8 & 0.78 & 13.4 & 10. & & 0.51 & 1232 & 3.39 & 13.52 & 25. & & 3.14 & 807 & 13.29 & 2.93 & 204 & & 31 & $<0.5$ & 8.59 \\
\hline M82 & 0.56 & 13.6 & 17. & & 0.89 & 1163 & 3.51 & 24.35 & 40. & & 8.35 & 569 & $<0.5$ & 4.43 & 160 & & 01 & 0.56 & 29.13 \\
\hline M86 & 0.53 & 17.3 & 15. & & 0.88 & 1072 & 1.88 & 27.05 & 44. & & 6.08 & 774 & $<0.5$ & 4.36 & 262 & & .87 & 0.98 & 25.78 \\
\hline M93 & 0.4 & 20.0 & 15. & & 0.72 & 1001 & $<0.5$ & 23.66 & 43. & & 6.09 & 968 & $<0.5$ & 5.05 & 210 & & .81 & $<0.5$ & 14.36 \\
\hline Sample No. & $\mathrm{Se}$ & $\mathrm{Sm}$ & $\mathrm{Sn}$ & $\mathrm{Sr}$ & $\mathrm{Ta}$ & $\mathrm{Tb}$ & $\mathrm{Te}$ & Th & $\mathrm{Ti}$ & $\mathrm{Tl}$ & $\mathrm{Tm}$ & $\mathrm{V}$ & $\mathrm{U}$ & W & $\mathrm{Y}$ & $\mathrm{Yb}$ & $\mathrm{Zn}$ & $\mathrm{Zr}$ & L.O.I \\
\hline M11 & 0.48 & 3.31 & 9.33 & 372 & 1.04 & 1.61 & 0.68 & 6.00 & 4134 & 1.15 & 0.56 & 174 & 2.92 & 3.58 & 13.84 & 2.34 & 75.08 & $\begin{array}{ll}8 & 61.01\end{array}$ & 2.61 \\
\hline M16 & 1.39 & 3.17 & 12.18 & 403 & 1.06 & 1.61 & 0.74 & 6.82 & 4007 & 1.46 & 0.56 & 162 & 3.70 & 7.60 & 18.23 & 2.58 & 114 & 51.44 & 5.58 \\
\hline M20 & 4.22 & 3.32 & 13.27 & 416 & 0.92 & 1.64 & 0.75 & 7.26 & 5103 & 1.75 & 0.56 & 229 & 4.14 & 8.31 & 17.34 & 2.63 & 81.77 & $7 \quad 49.98$ & 2.93 \\
\hline M22 & 5.18 & 5.04 & 13.92 & 362 & 0.84 & 1.42 & 0.66 & 7.44 & 5369 & 1.83 & 0.44 & 227 & 4.50 & 4.74 & 24.77 & 3.44 & 218 & 254 & 4.46 \\
\hline M25 & $<0.05$ & 3.50 & 14.13 & 333 & 0.77 & 1.41 & 0.65 & 5.94 & 3987 & 1.80 & 0.43 & 152 & 4.65 & 3.74 & 17.23 & 2.58 & 106 & 146 & 2.28 \\
\hline M26 & 3.28 & 3.68 & 12.95 & 395 & 0.79 & 1.48 & 0.66 & 6.23 & 4054 & 1.75 & 0.46 & 145 & 3.88 & 7.07 & 17.25 & 2.43 & 146 & 119 & 2.74 \\
\hline M31 & 1.80 & 4.42 & 12.54 & 291 & 1.13 & 1.73 & 0.80 & 8.27 & 5493 & 1.50 & 0.54 & 240 & 4.02 & 5.74 & 19.77 & 3.00 & 81.31 & $1 \quad 137$ & 2.95 \\
\hline M33 & 6.12 & 2.79 & 11.92 & 331 & 0.75 & 1.23 & 0.55 & 5.86 & 3325 & 1.41 & 0.38 & 105 & 3.85 & 7.36 & 19.21 & 2.50 & 150 & 154 & 3.31 \\
\hline M34 & 0.27 & 5.58 & 12.66 & 336 & 0.89 & 1.45 & 0.67 & 7.42 & 6574 & 1.53 & 0.44 & 135 & 3.70 & 3.48 & 39.44 & 4.61 & 104 & 427 & 2.63 \\
\hline M37 & 0.26 & 5.34 & 9.93 & 291 & 0.73 & 1.34 & 0.63 & 6.58 & 6128 & 1.18 & 0.39 & 127 & 3.16 & 1.98 & 37.39 & 4.39 & 83.96 & $\begin{array}{ll}5 & 327\end{array}$ & 1.19 \\
\hline M40 & 0.68 & 5.48 & 14.56 & 359 & 0.98 & 1.82 & 0.78 & 8.16 & 6564 & 1.86 & 0.52 & 254 & 4.62 & 7.35 & 21.88 & 3.32 & 76.71 & 1196 & 2.47 \\
\hline M41 & 0.04 & 4.40 & 10.41 & 284 & 0.65 & 1.02 & 0.47 & 5.55 & 2936 & 1.30 & 0.37 & 41.23 & 3.16 & 3.80 & 25.05 & 2.52 & 93.60 & 65.70 & 2.31 \\
\hline M43 & 0.78 & 4.73 & 10.79 & 237 & 0.53 & 1.03 & 0.47 & 5.02 & 2539 & 1.32 & 0.36 & 38.34 & 3.20 & 3.03 & 19.44 & 2.02 & 107 & 67.90 & 2.15 \\
\hline M48 & 0.05 & 3.86 & 16.02 & 568 & 1.13 & 1.99 & 0.86 & 8.28 & 4332 & 2.05 & 0.60 & 192 & 5.12 & 12.42 & 20.69 & 3.08 & 132 & 143 & 2.82 \\
\hline M5 & 4.93 & 3.44 & 10.78 & 391 & 1.12 & 1.89 & 0.82 & 8.10 & 4299 & 1.30 & 0.57 & 195 & 3.36 & 3.75 & 16.74 & 2.57 & 113 & 116 & 1.59 \\
\hline M50 & 0.04 & 4.64 & 8.86 & 498 & 0.66 & 1.27 & 0.54 & 5.02 & 3463 & 1.00 & 0.40 & 87.42 & 2.65 & $<0.5$ & 14.07 & 1.77 & 91.80 & 84.75 & 1.69 \\
\hline M53 & 0.43 & 4.85 & 15.30 & 554 & 1.17 & 2.04 & 0.88 & 8.10 & 4717 & 1.93 & 0.63 & 233 & 4.66 & 13.01 & 18.59 & 3.11 & 133 & 136 & 2.45 \\
\hline M55 & 0.29 & 5.04 & 11.34 & 441 & 0.83 & 1.63 & 0.68 & 6.60 & 3635 & 1.39 & 0.51 & 143 & 3.55 & 8.58 & 17.47 & 2.64 & 58.59 & 984.07 & 1.29 \\
\hline M60 & 2.47 & 2.83 & 11.34 & 408 & 0.77 & 1.23 & 0.60 & 5.28 & 3425 & 1.41 & 0.44 & 126 & 3.56 & 9.16 & 14.94 & 2.21 & 56.33 & 358.78 & 1.71 \\
\hline M63 & 0.04 & 3.45 & 8.95 & 343 & 0.44 & 1.03 & 0.41 & 3.90 & 2441 & 1.13 & 0.31 & 54.12 & 2.67 & 0.89 & 14.07 & 1.45 & 59.51 & 148.13 & 2.23 \\
\hline M69 & 0.04 & 3.78 & 10.03 & 626 & 0.62 & 1.44 & 0.59 & 4.75 & 3860 & 1.19 & 0.45 & 89.90 & 3.22 & 1.06 & 15.89 & 1.89 & 70.55 & $5 \quad 74.03$ & 2.45 \\
\hline M72 & 0.04 & 3.33 & 9.56 & 563 & 0.81 & 1.53 & 0.65 & 6.01 & 4364 & 1.13 & 0.51 & 126 & 3.11 & 3.64 & 17.25 & 2.53 & 122 & 196 & 1.6 \\
\hline M75 & 0.04 & 3.18 & 4.36 & 568 & 0.19 & 0.35 & 0.25 & 2.60 & 1730 & 0.63 & 0.19 & 13.62 & 1.34 & 2.52 & 8.61 & 0.83 & 71.94 & $4 \quad 59.65$ & 1.42 \\
\hline M76 & 4.58 & 4.03 & 8.33 & 803 & 0.81 & 1.40 & 0.63 & 6.54 & 4247 & 0.95 & 0.39 & 107 & 2.71 & 2.30 & 19.56 & 2.70 & 129 & 217 & 1.33 \\
\hline M78 & 0.04 & 2.52 & 8.18 & 525 & 0.65 & 1.09 & 0.49 & 5.11 & 3278 & 1.02 & 0.37 & 71.04 & 2.48 & $<0.5$ & 16.59 & 1.99 & 64.96 & 683.41 & 1.67 \\
\hline M8 & 2.77 & 4.80 & 6.53 & 379 & 0.48 & 0.96 & 0.45 & 5.16 & 3359 & 0.75 & 0.35 & 64 & 1.86 & $<0.5$ & 20.32 & 2.44 & 104 & 55.57 & 0.52 \\
\hline M82 & 2.30 & 3.75 & 13.70 & 299 & 1.21 & 2.17 & 0.89 & 8.43 & 5956 & 1.69 & 0.65 & 278 & 4.49 & 1.15 & 23.80 & 3.72 & 57.37 & $7 \quad 150$ & 3.34 \\
\hline M86 & 0.77 & 4.55 & 13.27 & 489 & 1.17 & 2.14 & 0.87 & 8.41 & 6637 & 1.50 & 0.64 & 214 & 4.49 & 5.77 & 32.56 & 4.39 & 89.86 & $6 \quad 237$ & 4.16 \\
\hline M93 & 6.17 & 5.71 & 8.85 & 390 & 0.94 & 1.75 & 0.72 & 7.60 & 5835 & 1.20 & 0.57 & 209 & 2.87 & $<0.5$ & 30.05 & 4.13 & 77.23 & $3 \quad 275$ & 1.03 \\
\hline
\end{tabular}


charts [38], are within andesite, dacite and rhyodacite (Figure 16). For determining the magmatic series of volcanic rocks, Irvina \& Baragar charts [39] were used and samples were within sub-alcaline, calc-alkaline and rarely tholeiitic because of weathering and lose of potassium oxide (Figure 17 and Figure 18). According to the Luba charts [40] (Figure 19) region rocks are sub-alcaline and

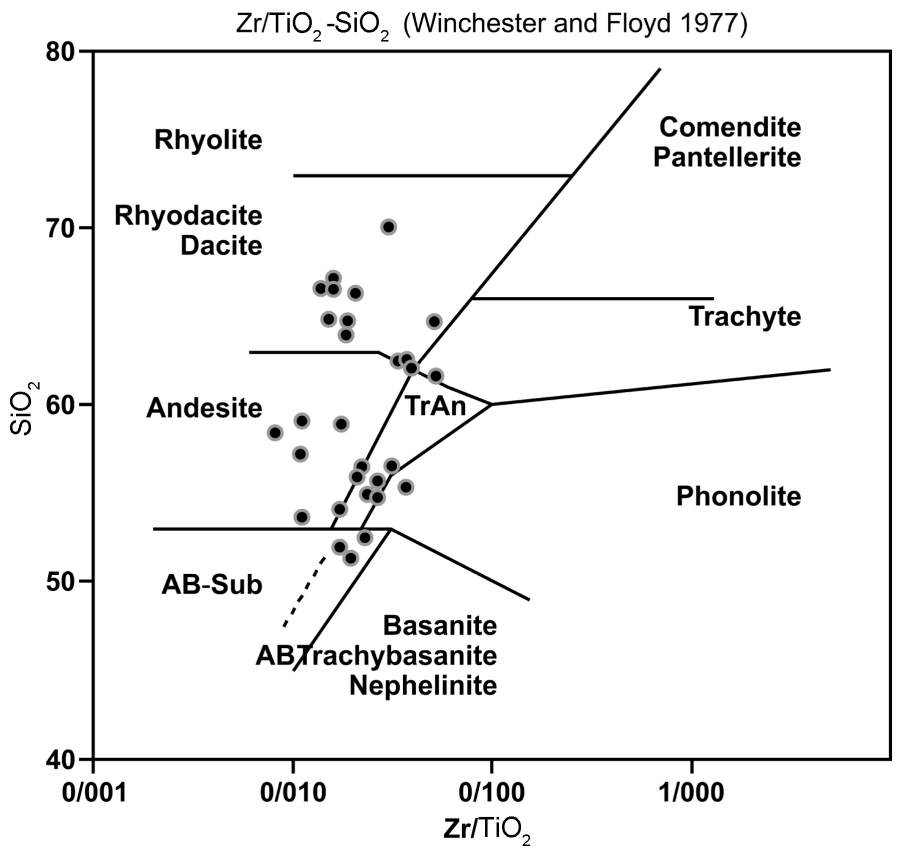

Figure 16. Location of region samples in volcanic rocks nomination charts by using $\mathrm{SiO}_{2}, \mathrm{Zr} / \mathrm{TiO}_{2}$.

\section{AFM plot (Irvine and Baragar 1971)}

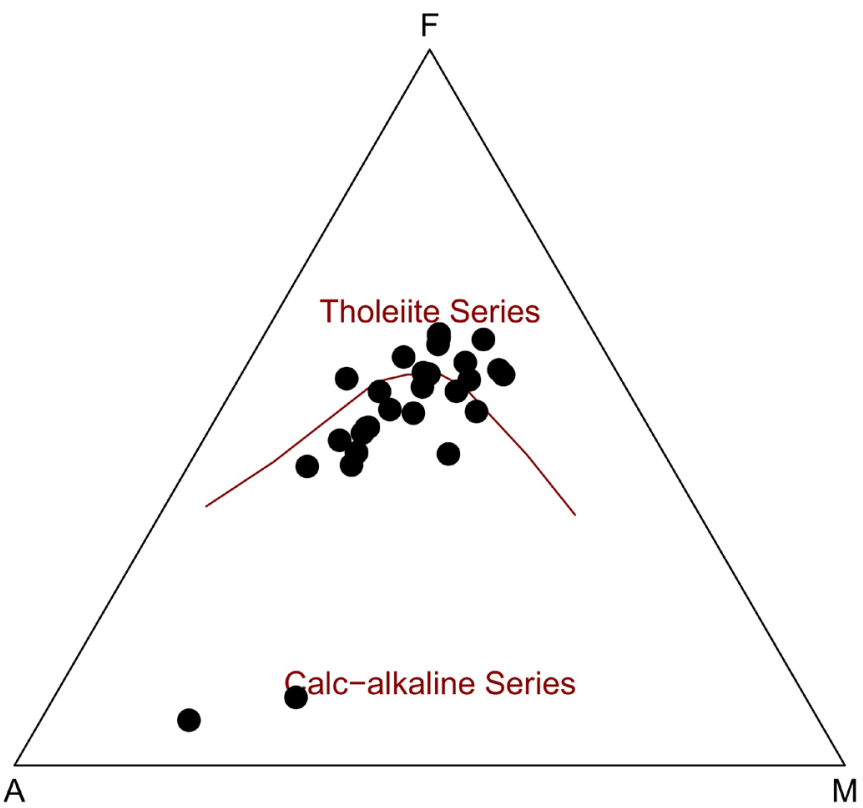

Figure 17. $\mathrm{FeO}^{*}-\left(\mathrm{Na}_{2} \mathrm{O}+\mathrm{K}_{2} \mathrm{O}\right)-\mathrm{MgO}$ for separation of tholeiitic and calc-alkaline range (Irvina \& Baragar, 1971). 


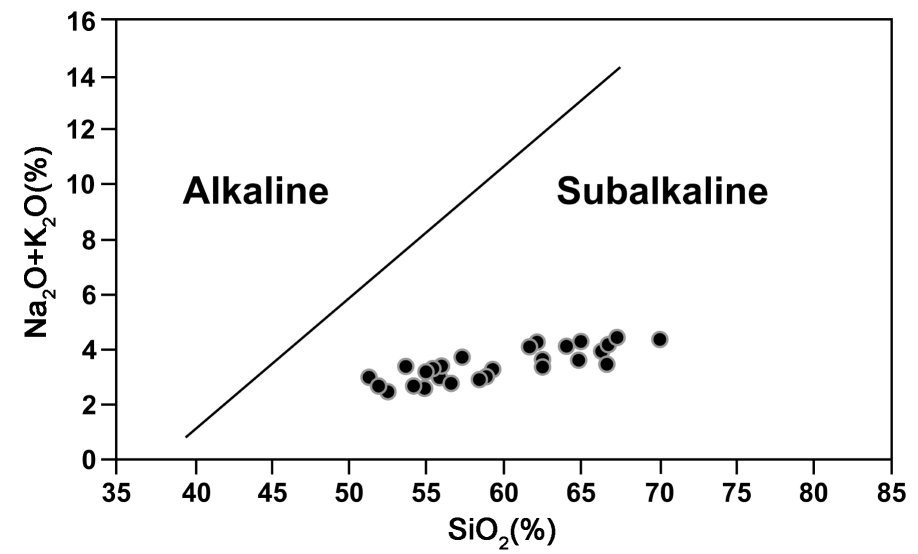

Figure 18. Samples location in the volcanic rocks segmentation chart to alkaline and sub-alkaline according to the total alkali versus silica (Irvina \& Baragar, 1971).

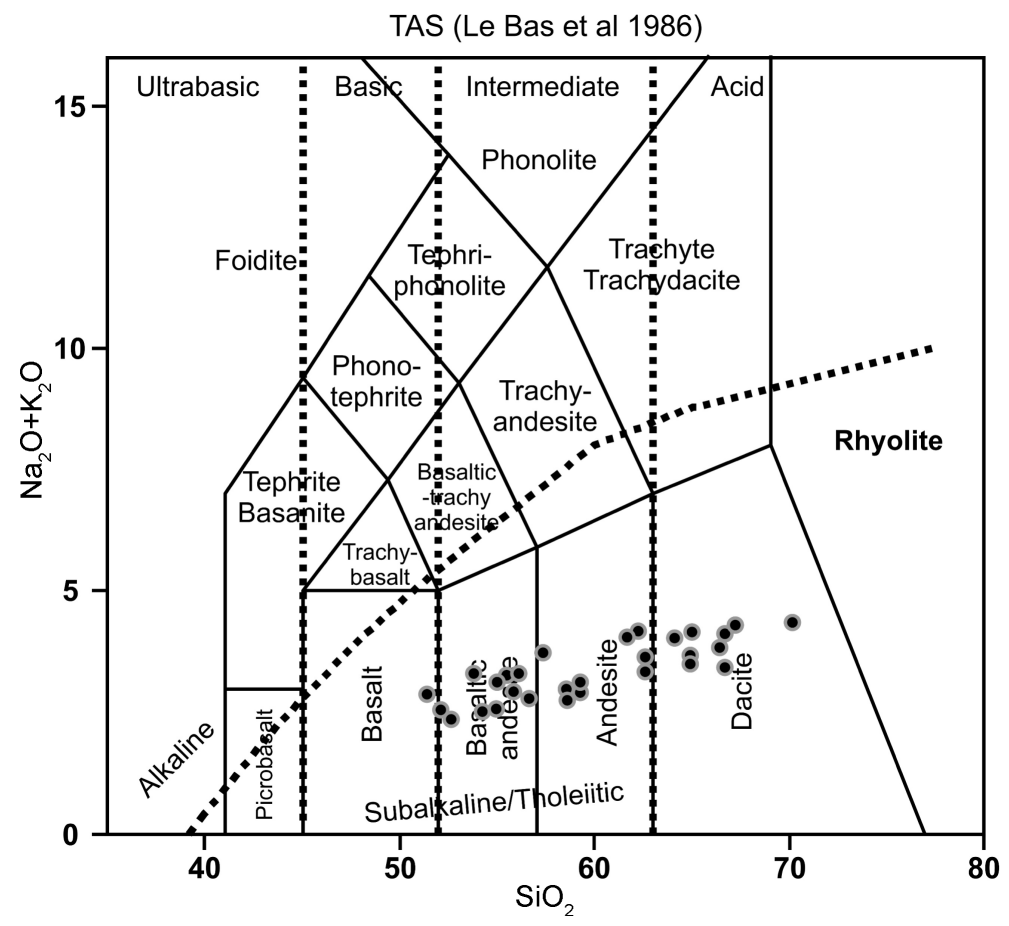

Figure 19. Location of studied samples in volcanic rocks nomination chart according to the TAS method (Le Bas, 1986).

within andesite, basaltic andesite basaltic and dacite. According to the $\mathrm{K}_{2} \mathrm{O}-\mathrm{SiO}_{2}$ (Figure 20) [41] most of the region volcanic rocks are in intermediate groups and fewer in acid range. All of the samples are in low-potassium tholeiitic range.

\subsubsection{Magmatic Tectonic Pattern}

For recognizing tectonic environment of volcanic rocks within studied area different charts were used. According to the Muller \& Groves (1977) [42] charts all samples are in range of volcanic rocks of volcanic arcs after collision (Figure 21 and Figure 22). Moein Vaziri [7] believes that Neotethys Ocean due to movement of afro-arabian plate towards the northeast has subducted (drived) to the 


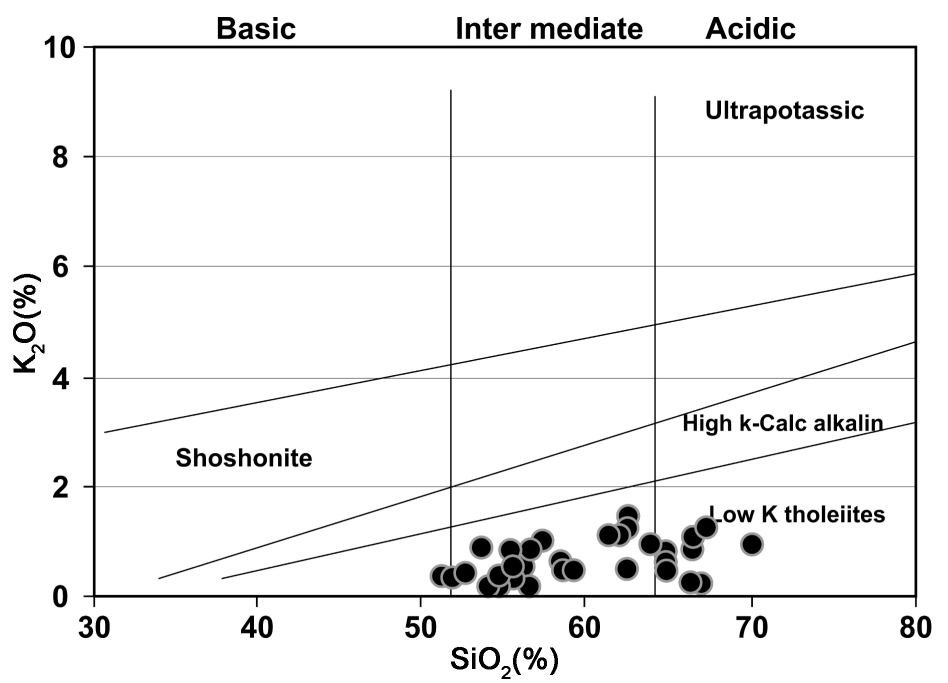

Figure 20. Changes in $\mathrm{SiO}_{2}, \mathrm{~K}_{2} \mathrm{O}$ in volcanic rocks of studied region (Muller et al., 1998).

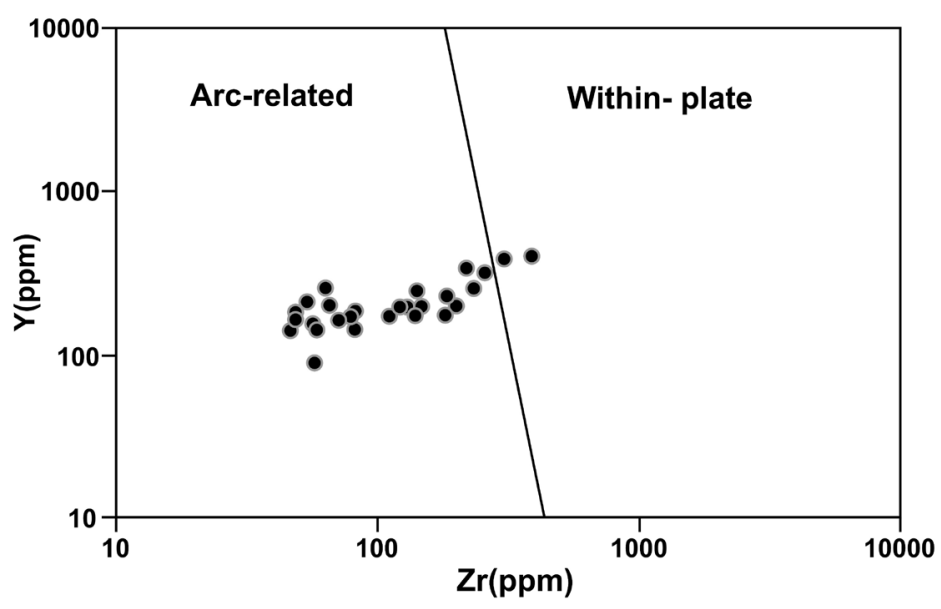

Figure 21. Y/Zr diagram for determination of tectonic setting of volcanic rocks of studied area (Muller and Groves, 1977).

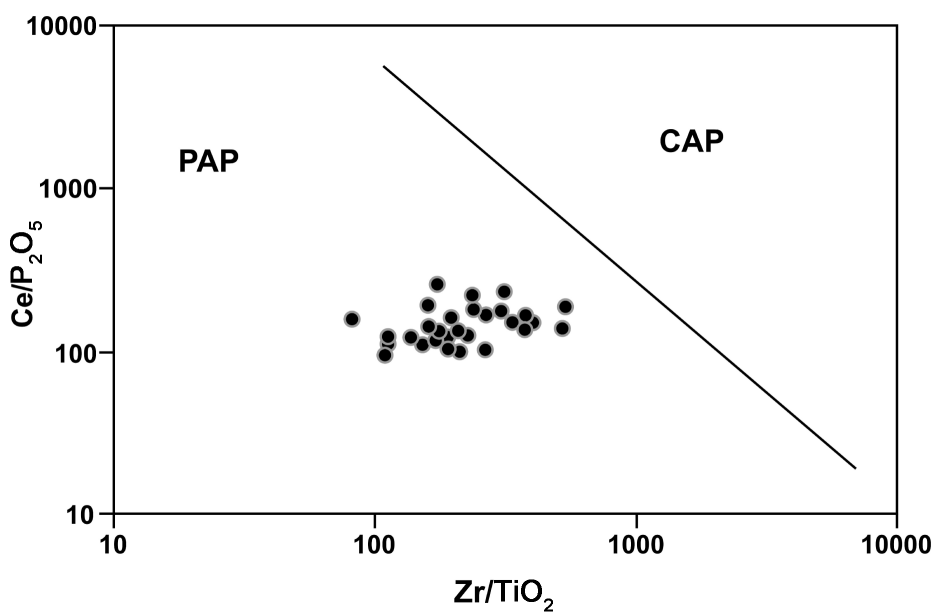

Figure 22. $\mathrm{Zr} / \mathrm{TiO}_{2}-\mathrm{Ce} / \mathrm{P}_{2} \mathrm{O}_{5}$ diagtam (Muller and Groves, 1977) for separation of active continental margin magmatic arc (CAP) and magmatic arc after hitting (PAP). 
continent of Central Iran. The matching of Subduction zones and dealing with old hydrothermal blades which had created Mesozoic rift within the continent, could cause disturbance in classical magmatism in mentioned tectonic setting. A break of Neotethys oceanic crust in the upper Cretaceous allows that depressed and stopped part of the shell get uniformly warm (hot) and causes the creation of severe volcanoes in the Eocene period. These phenomena continued with less intensity in other parts of the Tertiary [1]. Some researchers [1] [21] believed that most intrusive and semi-volcanic masses of Iran's tertiary are characterized by Oligo-Miocene ages which formed as result of pressure relief deep magmatic reservoirs of Eocene volcano in orogenic period of Pyrenees and astrains era [7].

In Figure 23, distribution pattern of rare earth elements (REE) in region samples has been ruled against chondrites [43]. According to the diagrams, all samples are richer than chondrites in REE. Among these, Light rare earth elements (LREE) are richer than High rare earth elements (HREE). As seen in figure, behavior of Eu is almost like other REE and do not represent any particular anomaly. Rollinson, [44] mentioned that, this phenomena happens when fugacity of oxygen is high and distribution coefficient of Eu is low. In spider diagram normative to MORB [45], region rocks show somewhat depletion in HFSE elements (Figure 24), While enriched from $\mathrm{K}$, Th and $\mathrm{Rb}$ elements which has features of magmatic arc rocks or volcanic arc rocks [44]. These elements are special for crustal rocks and may indicate the digestion of crustal rocks by the initial molten material.

In subduction area, fluid released from the upper part of subducted lithosphere which is rich of LILE and poor of $\mathrm{Nb}$ are added to the mantle wedge [46].

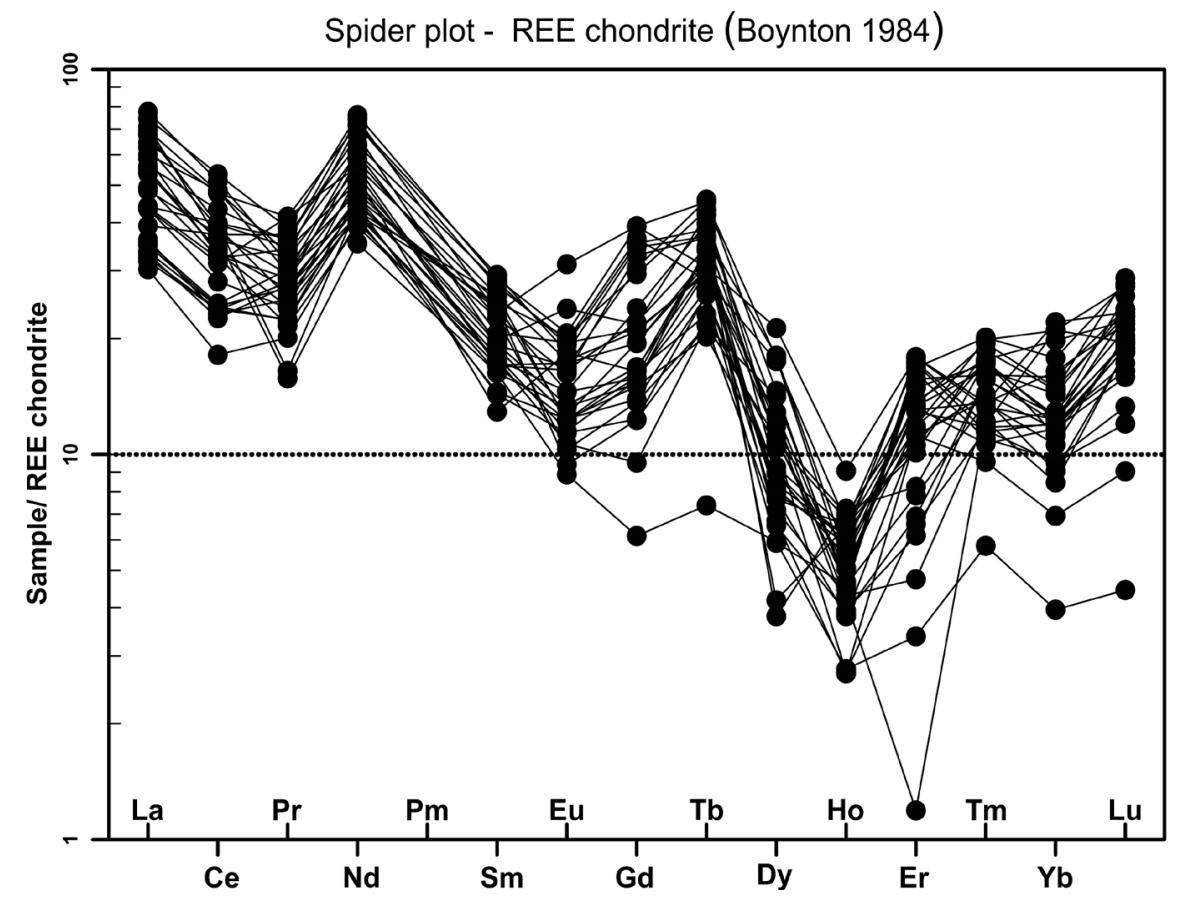

Figure 23. The normative pattern of rare earth elements compared to chondrites in studied samples (Boyton, 1984). 


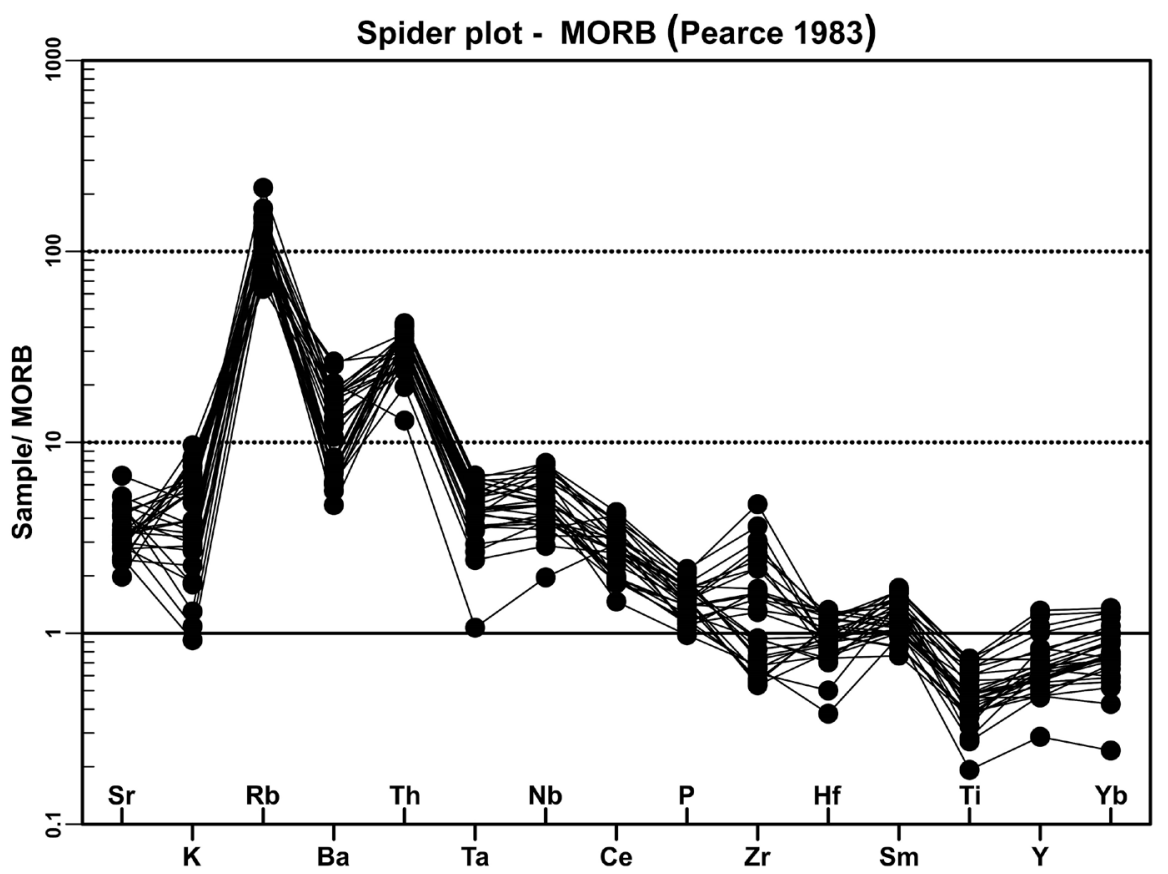

Figure 24. Spider diagram normalized to MORB in studied area (Pearce, 1983).

The high amount of $\mathrm{Ce}, \mathrm{K}, \mathrm{Rb}$ and $\mathrm{Ba}$ elements in these rocks can indicate: 1) Digestion continental crust rocks in the direction of the upper mantle and molten rock is swept; 2) The involvement of magmatic origin of continental crust (partial melting) or deeper magmatic origin (magma mixing); 3) The effect of alkaline metasomatism on the area rocks during or after crystallization and positioning the rocks and 4) Extensive hydrothermal alteration in area rocks (kaolinisation with quartz-sericite alteration) which is a result of magmatic fluids rich shell elements.

In the primitive mantle normalized charts (Figure 25) Enrichment from LREE and HREE were observed in all samples. Enrichment of LREE elements is higher than HREE and negative gradient of the graph indicates the higher amount of LREE than HREE that conforms with the overall pattern of spider diagrams subduction zones. Defant \& Drummond [47] mentioned partial enrichment of $\mathrm{P}$ in basaltic and andesite samples caused due to presence high amount of apatite in these samples. Provatke and Klemme [48] believed that presence of apatite in rocks in addition to creating a positive anomaly in $\mathrm{P}$ can cause $\mathrm{Sr}$ enrichment in rocks. Sr plays as a highly consistent element in apatite. Positive anomaly of $\mathrm{Rb}$ May be happened due to contamination magma with crustal (due to the high concentration of these elements in the continental crust). Because of the continental crust enriched with $\mathrm{Nb}$ and $\mathrm{Ta}$, so suspension of these rocks magmas (although short-term), in the lower continental crust with penetration of crustal fluids into the magma or by digestion of crustal magma rocks cause this type of anomaly in studied samples [49] [50].

In conjunction with enriched potassium relative to primitive mantle, Watson [51] believed that contamination of magma with continental crust, Even if the other elements remain unchanged, significant amounts of potassium finds a way 
into the basaltic magma. Also the positive anomaly of $\mathrm{Sr}$ in basaltic rocks is justified by the presence in these rocks plagioclase [52]. Similar and parallel trends drawn in all spider diagrams can introduce a single source for these rocks.

Meanwhile, negative anomalies of Ti element in lava can be formed one hand to participate in the partial melting of lithospheric mantle linked metasomatized and on the other hand crustal contamination resulting from digestion are attributed zinocryst [44].

Using $\mathrm{SiO}_{2}$ alternation plot against $\mathrm{Sr} / \mathrm{Y}$ [53] (Figure 26) volcanic rocks of studied area are not located in adakitic rocks groups. Also the $\mathrm{Y}$ against $\mathrm{Sr} / \mathrm{Y}$ diagram [47] (Figure 27) indicate location samples within the volcanic arc.

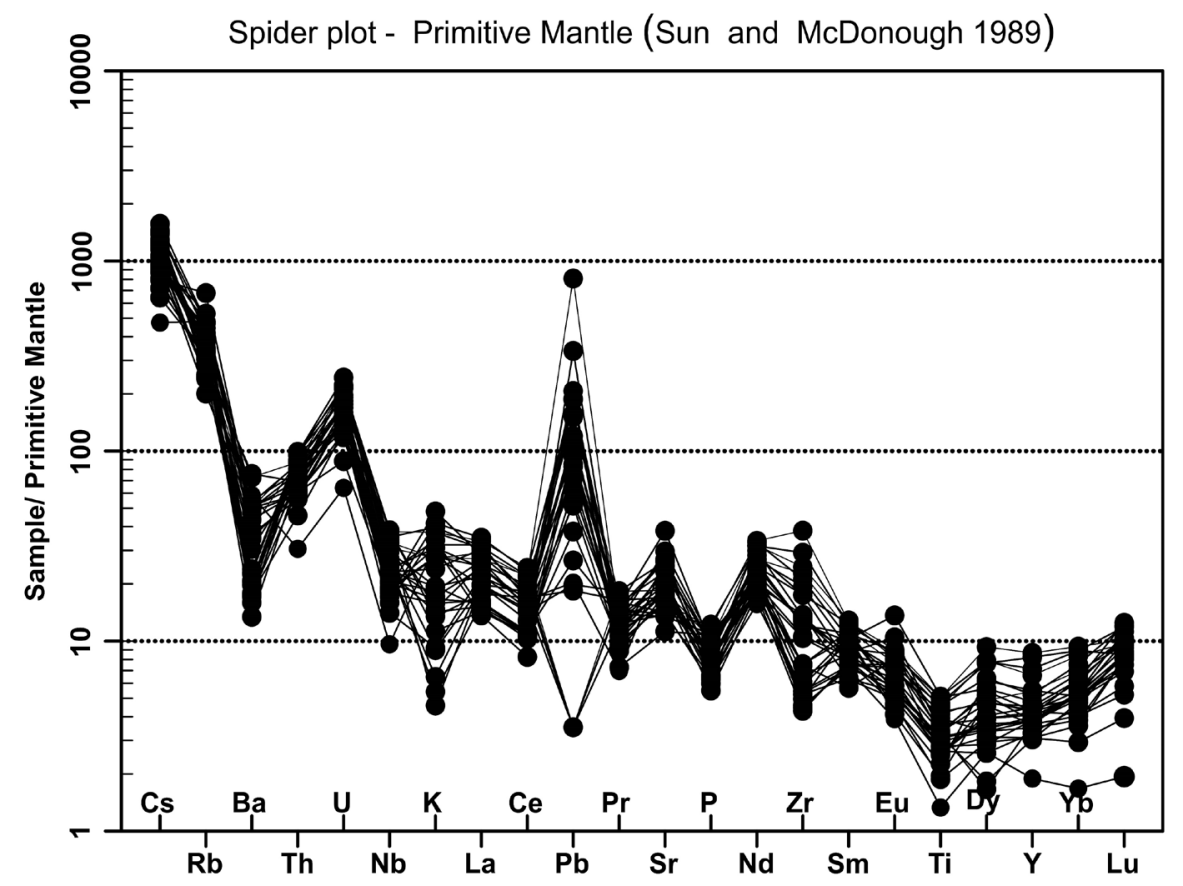

Figure 25. Spider diagram normalized to primitive mantle (Sun \& McDonough, 1989).

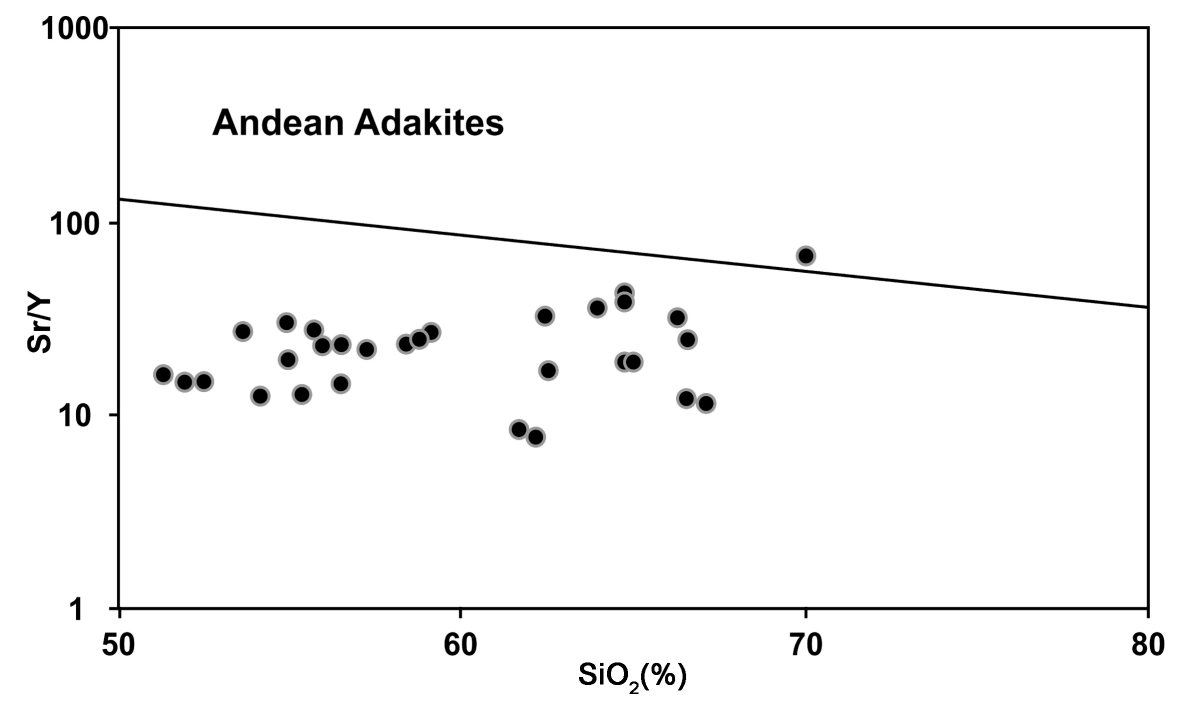

Figure 26. $\mathrm{SiO}_{2} / \mathrm{Sr} / \mathrm{Y}$ diagram (Yofodzinski et al., 2001). 


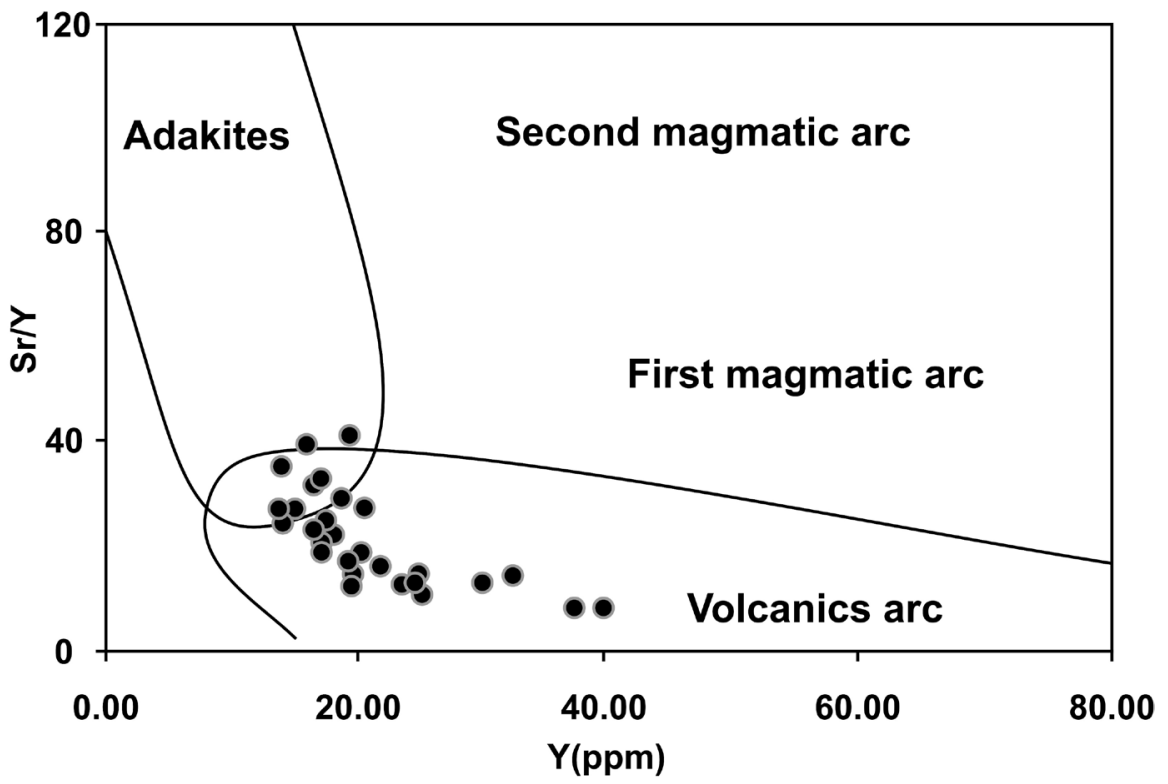

Figure 27. Y alternation against Sr/Y (Defant \& Drommond, 1990).

\section{Conclusion}

Lithological composition of volcanic rocks in studied area consists lava basalt, andesite and dacite. The most abundant volcanic rock outcrop is andesite. The dominant texture of these rocks is porphyric with microcrystalline pulp. The magmatic nature of sub-alcaline volcanic rocks is generally alkaline and less tholeiitic. The region rocks enriched with LILE and HFSE elements were less depleted in comparison to MORB which depends on volcanic arcs. Volcanic rocks of studied area are located in tectonic setting of volcanic arc after the collision (PAP). Also presence of negative anomaly in $\mathrm{P}, \mathrm{Nb}, \mathrm{Ta}$ and $\mathrm{Ti}$ elements could indicate the formation of rocks in subduction zone. According to the $\mathrm{Zr} / \mathrm{Y}$ diagram, all samples are located in extent of volcanic arc. Volcanic rocks of region are not in adactic rock groups. The tectonic evidence of region shows that Neogene magmatism of the region is connected with a collision area with Tethys Ocean subduction under Magmatic Belt of CIVB and collision between the Arabic plate central Iran during the Neogene era.

\section{References}

[1] Agha Nabati, A. (2004) Iran's Geology. Geological survey of Iran, Tehran, 586 p.

[2] Farhoudi, G. (1978) A Comparison of Zagros Geology to Island Arcs. The Journal of Geology, 86, 323-334.

[3] Emami, M.H. (1981) Géologie de la région de Qom-Aran (Iran): Contribution a l'étude dynamique et géochimique du volcanisme Te rtiaire de l'I-ran Central. Ph.D. Thèse, Sciences naturelles Univ. Sc. Et Medicale de Grenoble., 489 p.

[4] McQuarrie, N. (2004) Crustal Scale Geometry of the Zagros Fold-Thrust Belt, Iran. Journal of Structural Geology, 26, 519-535.

[5] Jung, D., Kursten, M. and Tarakian, M. (1976) Post-Mesozoic Volcanism in Iran and Its Relation to the Sub-Duction of the Afro-Arabian under the Eurasian Plate. In: Pilger, A. and Rosler, A., Eds., Afar between Continental and Oceanic Rifting, 
Volume II, Stuttgart, Schweizerbatsche Verlagsbuch-Handlung, 175-181.

[6] Berberian, F., Muir, I.D., Pankhurst, R.J. and Berberian, M. (1982) Late Cretaceous and Early Miocene Andean-Type Plutonic Activity in Northern Makran and Central Iran. Journal of the Geological Society, 139, 605-614. https://doi.org/10.1144/gsigs.139.5.0605

[7] Moeenvaziri, H. (1998) Magmatism in Iran, Tehran. Tarbiat Moalem University.

[8] Boomeri, M., Nakashima, K. and Lentz, D.R. (2010) The Sarcheshmeh Porphyry Copper Deposit, Kerman, Iran: A Mineralogical Analysis of the Igneous Rocks and Alteration Zones Including Halogen Element Systematics Related to $\mathrm{Cu}$ Mineralization Processes. Ore Geology Reviews, 38, 367-381.

[9] Hosseini, M., Zarasvandi, A.R. and Liaghat, S. (2010) Tectonic Setting and Petrogenesis of Darreh-Zereshk Granitoids (SW Yazd, Iran) and Comparison with Some World Skarn Granitoids. Abstract for the 1st International Applied Geological Congress, Department of Geology, Islamic Azad University-Mashad Branch, Iran, 26-28 April 2010.

[10] Alavi, M. (1994) Tectonics of the Zagros Orogenic Belt of Iran: New Data and Interpretations. Tectonophysics, 229, 211-238.

[11] Shahabpour, J. (2005) Tectonic Evolution of the Orogenic Belt in the Region Located between Kerman and Neyriz. Journal of Asian Earth Sciences, 24, 405-417.

[12] Barzegar, H. (2007) Geology, Petrology and Geochemical Characteristics of Alteration Zones within the Seridune Prospect, Kerman, Iran. Ph.D. Thesis, Georessourcen und Material technik der Rheinisch Westfälischen Technischen Hochschule Aachen, 202 p.

[13] Kirkham, R.V. and Dunne, K.P.E. (2000) World Distribution of Porphyry, Porphyry-Associated Skarn, and Bulk-Tonnage Epithermal Deposits and Deposits. Geological Survey of Canada Open File Report 3792a, 26 p.

[14] Walker, R. and Jackson, J. (2002) Offset and Evolution of the Gowk fault, S.E. Iran: A Major Intra-Continental Strike-Slip System. Journal of Structural Geology, 24, 1677-1698.

[15] McInnes, B.I.A., Evans, N.J., Belousova, E., Griffin, W.T. and Andrew, R.L. (2003) Timing of Mineralization and Exhumation Processes at the Sarcheshmeh and Meiduk Porphyry Cu Deposits, Kerman Belt, Iran: Mineral Exploration and Sustainable Development. Millpress, Rotterdam, 1197-1200.

[16] Sillitoe, R.H. (1997) Characteristics and Controls of the Largest Porphyry CopperGold and Epithermal Gold Deposits in the Circum-Pacific Region. Australian Journal of Earth Sciences, 44, 373-388.

[17] Titley, S.R. (1982) Advances in Geology of the Porphyry Cu Deposits. University of Arizona Press, Tucson, $560 \mathrm{p}$.

[18] Ghorbani, M. (2013) The Economic Geology of Iran: Mineral Deposits and Natural Resources. Springer Science Business Media, Dordrecht, 581 p. https://doi.org/10.1007/978-94-007-5625-0

[19] Sawkins, F.J. (1990) Metal Deposits in Relation to Plate Tectonics. Springer-Verlag, Berlin, 461 p. https://doi.org/10.1007/978-3-662-08681-0

[20] Ghadami, G., Shahre-Babaki, A.M. and Mortazavi, M. (2008) Post-Collisional PlioPleistocene Adakitic Volcanism in Central Iranian Volcanic Belt: Geochemical and Geodynamic Implications. Journal of Sciences Islamic Republic of Iran, 19, 223-235.

[21] Berberian, M. and King, G.C.P. (1981) Towards a Paleogeography and Tectonic Evolution of Iran. Canadian Journal of Earth Sciences, 18, 210-265.

https://doi.org/10.1139/e81-019 
[22] Dercourt, J., Ricou, L.E. and Vrielynck, B., Eds. (1993) Atlas Tethys, Palaeoenvironmental Maps: Explanatory Notes. Gauthier-Villars, Paris, 14 Maps, 1 Plate, 307 p.

[23] Ricou, L.E. (1994) Tethys Reconstructed: Plates Continental Fragments and Their Boundaries Since $260 \mathrm{Ma}$ from Central America to South-Eastern Asia. Geodinamica Acta, 7, 169-218. https://doi.org/10.1080/09853111.1994.11105266

[24] Mohajjel, M., Fergusson, C.L. and Sahandi, M.R. (2003) Cretaceous-Tertiary Convergence and Continental Collision, Sanandaj-Sirjan Zone, Western Iran. Journal of Asian Earth Sciences, 21, 397-412.

[25] McClay, K.R., Whitehouse, P.S., Dooley, T. and Richards, M. (2004) 3D Evolution of Fold and Thrust Belts Formed by Oblique Convergence. Marine Geology, 21, 857-877.

[26] Agard, P., Omrani, J., Jolivet, L. and Mouthereau, F. (2005) Convergence History across Zagros (Iran): Constraints from Collisional and Earlier Deformation. International Journal of Earth Sciences, 94, 401-419. https://doi.org/10.1007/s00531-005-0481-4

[27] Bonatti, E. (1987) Rifting or Drifting in the Red Sea? Nature, 330, 692-693. https://doi.org/10.1038/330692a0

[28] Hempton, M.R. (1987) Constraints on Arabian Plate Motion and Extensional History of the Red Sea. Tectonics, 6, 687-705. https://doi.org/10.1029/TC006i006p00687

[29] Guiraud, R. and Bosworth, W. (1997) Senonian Basin Inversion and Rejuvenation of Rifting in Africa and Arabia: Synthesis and Implications to Plate-Scale Tectonics. Tectonophysics, 282, 39-82.

[30] McClusky, S., Balassanian, S., Barka, A., Demir, C., Ergintav, S., Georgiev, I., Gurkan, O., Hamburger, M., Hurst, K., Kahle, H., Kastens, K., Kekelidze, G., King, R., Kotzev, V., Lenk, O., Mahmoud, S., Mishin, A., Nadariya, M., Ouzounis, A., Paradissis, D., Peter, Y., Prilepin, M., Reilinger, R., Sanli, I., Seeger, H., Tealeb, A., Toksoz, M.N. and Veis, G. (2003) Global Positioning System Constraints on Plate Kinematics and Dynamics in the Eastern Mediterranean and Caucasus. Journal of Geophysical Research: Solid Earth, 105, 2695-5719.

[31] Emami, M. and Hajian, J. (1991) Geology Map of Qom (Scale: 1:250,000). Geological Survey of Iran.

[32] Alaee Mahabadi, S. (2001) Geology Map of Salafchegan-Khorhe (Scale: 1:100,000). Geological Survey of Iran.

[33] Hajian, J. (1999) Geology Map of Tafresh. Scale: 1:100000. Geological Survey of Iran.

[34] Monsef, R. (2011) Geochemistry and Petrogenesis of Neogen Volcanic and Sub Volcanic Rocks from West of Salafchegan to North of Delijan.

[35] Fowler, A.D. and Ach, D.E. (1996) A Model and Simulation of Branching Mineral Growth from Cooling Contacts and Glasses. Mineralogical Magazine, 60, 595-601. https://doi.org/10.1180/minmag.1996.060.401.05

[36] Shelly, D. (1993) Igneous and Metamorphic Rocks under the Microscope. Chapman $\&$ Hall, London, $445 \mathrm{p}$.

[37] Cox, K.G., Bell, J.D. and Pankhurts, R.J. (1979) The Interpretation of Igneous Rocks. George Allen and Unwin, London, 450 p. https://doi.org/10.1007/978-94-017-3373-1

[38] Winchester, J.A. and Floyd, P.A. (1977) Geochemical Discrimination of Different Magma Series and Their Differentiation Products Using Immobile Elements. Che- 
mical Geology, 20, 325-343.

[39] Irvina, T. and Baragar, W.R.A. (1971) A Guide to the Chemical Classification of the Common Volcanic Rocks Canadian. Journal of Earth Science Letters, 8, 523-548.

[40] Le Bas, M.J., Le Maitre, R.W., Streckeisen, A. and Zanettin, B. (1986) A Chemical Classification of Volcanic Rocks Based on the Total Alkali-Silica Diagram. Journal of Petrology, 27, 745-750. https://doi.org/10.1093/petrology/27.3.745

[41] Muller, A., et al. (1998) New Aspects of Electron Transfer Revealed by the Crystal Structure of a Truncated Bovine Adrenodoxin, Adx(4-108). Structure, 6, 269-280. https://doi.org/10.1016/S0969-2126(98)00031-8

[42] Muller, D. and Groves, D.I. (1997) Potassic Igneous Rocks and Associated GoldCopper Mineralization. Springer-Verlag, Berlin, Heidelberg, 238 p.

[43] Boynton, W.V. (1984) Cosmochemistry of the Rare Earth Elements: Meteorite Studies. In: Henderson, P., Ed., Developments in Geochemistry, Elsevier Sci. Publ. Co., Amsterdam, 63-114.

[44] Rollinson, H. (1993) Using Geochemical Data: Evaluation, Presentation, Interpretation. Lonmman, London, $352 \mathrm{p}$.

[45] Pearce, J.A. (1983) Role of the Sub-Continental Lithosphere in Magma Genesis at Active Continental Margins. In: Hawkesworth, C.J. and Norry, M.J., Eds., Continental Basalts and Mantle Xenoliths, Shiva Cheshire, UK, 230-249.

[46] Borg, G. and Shackleton, RM. (1997) The Tanzania and NE Zaire Cratons. In: de Wit, M.J. and Ashwal, L.D., Eds., Greenstone Belts, Clarendon Press, Oxford, 608619.

[47] Defant, M.J. and Drummond, M.S. (1990) Derivation of Some Modern Arc Magmas by Melting of Young Subducted Lithosphere. Nature, 347, 662-665.

https://doi.org/10.1038/347662a0

[48] Provatke, S. and Klemme, S. (2006) Trace Element Partitioning between Apatite and Silicate Melts. Geochimica et Cosmochimica Acta, 70, 4513-4527.

[49] Wu, B.-M., Jahn, F.-Y., Wilde, S.A., Lo, C.-H., Yui, T.-F., Lin, Q., Ge, W.-C. and Sun, D.-Y. (2003) Highly Fractionated I-Type Granites in NE China (II): Isotopic Geochemistry and Implications for Crustal Growth in the Phanerozoic. Lithos, 67, 191-204.

[50] Wang, Y., Bryan, C., Xu, H. and Gao, H. (2003) Nanogeochemistry: Geochemical Reactions And Mass Transfers in Nanopores. Geology, 31, 387-390. https://doi.org/10.1130/0091-7613(2003)031<0387:NGRAMT>2.0.CO;2

[51] Watson, E.B. and Harrison, M. (1983) Zircon Saturation Revisited: Temperature and Composition Effects in a Variety of Crustal Magma Types. Earth and Planetary Science Letters, 64, 295-304.

[52] Moghazi, A.M. (2003) Geochemistry and Petrogenesis of a High-K Calc-Alkaline Dokhan Volcanic Suite, South Safaga Area, Egypt: The Role of Late Neoproterozoic Crustal Extension. Precambrian Research, 125, 161-178.

[53] Yogodzinski, G.M., Lees, J.M., Churikova, T.G., Dorendorf, F., Woerner, G. and Volynets, O.N. (2001) Geochemical Evidence for the Melting of Subducting Oceanic Lithosphere at Plate Edges. Nature, 409, 500-504. https://doi.org/10.1038/35054039 
Submit or recommend next manuscript to SCIRP and we will provide best service for you:

Accepting pre-submission inquiries through Email, Facebook, LinkedIn, Twitter, etc. A wide selection of journals (inclusive of 9 subjects, more than 200 journals)

Providing 24-hour high-quality service

User-friendly online submission system

Fair and swift peer-review system

Efficient typesetting and proofreading procedure

Display of the result of downloads and visits, as well as the number of cited articles Maximum dissemination of your research work

Submit your manuscript at: http://papersubmission.scirp.org/

Or contact ojg@scirp.org 